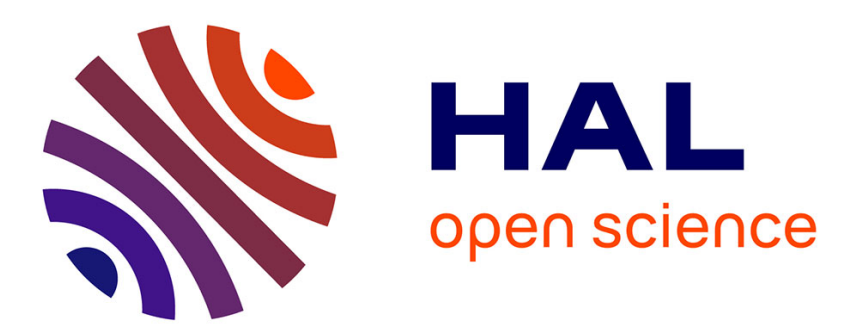

\title{
Reduction of CO2 by Hydrosilanes in the Presence of Formamidinates of Group 13 and 12 Elements
}

Weiheng Huang, Thierry Roisnel, Vincent Dorcet, Clement Orione, Evgueni Kirillov

\section{- To cite this version:}

Weiheng Huang, Thierry Roisnel, Vincent Dorcet, Clement Orione, Evgueni Kirillov. Reduction of $\mathrm{CO} 2$ by Hydrosilanes in the Presence of Formamidinates of Group 13 and 12 Elements. Organometallics, 2020, 39 (5), pp.698-710. 10.1021/acs.organomet.9b00853 . hal-02531302

HAL Id: hal-02531302 https://hal-univ-rennes1.archives-ouvertes.fr/hal-02531302

Submitted on 15 Apr 2020

HAL is a multi-disciplinary open access archive for the deposit and dissemination of scientific research documents, whether they are published or not. The documents may come from teaching and research institutions in France or abroad, or from public or private research centers.
L'archive ouverte pluridisciplinaire HAL, est destinée au dépôt et à la diffusion de documents scientifiques de niveau recherche, publiés ou non, émanant des établissements d'enseignement et de recherche français ou étrangers, des laboratoires publics ou privés. 
Reduction of $\mathrm{CO}_{2}$ by Hydrosilanes in the Presence of Formamidinates of Groups 13 and 12

\section{Elements}

Weiheng Huang, ${ }^{a}$ Thierry Roisnel, ${ }^{b}$ Vincent Dorcet, ${ }^{b}$ Clement Orione, ${ }^{c}$ and Evgueni Kirillov ${ }^{a,}$

${ }^{\text {a }}$ Organometallics: Materials and Catalysis laboratories, Univ Rennes, CNRS, ISCR (Institut des Sciences Chimiques de Rennes), UMR 6226, F-35700 Rennes, France

${ }^{\mathrm{b}}$ Centre de diffraction X, Univ Rennes, CNRS, ISCR (Institut des Sciences Chimiques de Rennes), UMR 6226, F-35700 Rennes, France

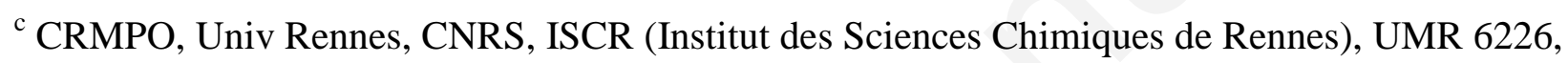
F-35700 Rennes, France

\section{Graphical Abstract / For the Table of content entry}

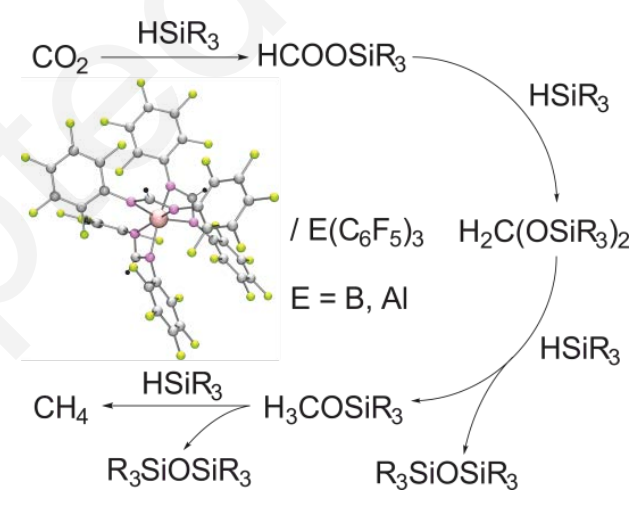

* Correspondence to Evgueni Kirillov (evgueni.kirillov@univ-rennes1.fr); Fax: +33 (0)223236938. 


\section{ABSTRACT}

Homoleptic complexes 1-M of groups 13 and 12 elements $(\mathrm{M}=\mathrm{B}-\mathrm{In}$ and $\mathbf{M}=\mathrm{Zn}$, respectively) incorporating electron-withdrawing formamidinate ligands $\left\{\left(\mathrm{C}_{6} \mathrm{~F}_{5}\right) \mathrm{N}=\mathrm{CH}-\mathrm{N}\left(\mathrm{C}_{6} \mathrm{~F}_{5}\right)\right\}^{-}$ $\left(\{\mathrm{NCN}\}^{-}\right)$were synthesized and isolated in high yields. The compounds were characterized by X-ray crystallography, NMR spectroscopy and elemental analysis. While single-component 1-M appeared to be sluggishly active or inactive in reduction of $\mathrm{CO}_{2}$ with hydrosilanes, a good catalytic performance was achieved with the two-component systems derived from combinations of $\mathbf{1 - M}$ and $\mathrm{E}\left(\mathrm{C}_{6} \mathrm{~F}_{5}\right)_{3}(\mathrm{E}=\mathrm{B}, \mathrm{Al})$. In particular, the binary combination 1-Al/B $\left(\mathrm{C}_{6} \mathrm{~F}_{5}\right)_{3}$ showed the best performance within the whole series, thus providing quantitative hydrosilane $\left(\mathrm{Et}_{3} \mathrm{SiH}\right)$ conversions under a range of conditions ( $\mathrm{P}_{\mathrm{CO} 2}$, temperature, benzene or bromobenzene solvents) and affording mainly $\mathrm{CH}_{2}\left(\mathrm{OSiEt}_{3}\right)_{2}$ and $\mathrm{CH}_{4}$ as products. Kinetic and mechanistic studies revealed that at the initiation step the binary catalytic systems undergo a complex transformation in the presence of $\mathrm{CO}_{2} / \mathrm{Et}_{3} \mathrm{SiH}$ affording the products of 1-Al decomposition, namely, $\left(\mathrm{C}_{6} \mathrm{~F}_{5}\right) \mathrm{N}(\mathrm{H}) \mathrm{SiEt}_{3}$, $\left(\mathrm{C}_{6} \mathrm{~F}_{5}\right) \mathrm{N}(\mathrm{Me}) \mathrm{SiEt}_{3},\{\mathrm{NCN}\}-\mathrm{SiEt}_{3}$ and also some unidentified aluminum species. Thus, the overall process of the reduction of $\mathrm{CO}_{2}$ with hydrosilanes is presumed to be catalyzed by complex multi-site systems, evolved from the formamidinate precursor 1-Al, implicating different tandem combinations of $N$-base $/ \mathrm{B}\left(\mathrm{C}_{6} \mathrm{~F}_{5}\right)_{3}$ with putative $\mathrm{Al}$-containing species.

\section{INTRODUCTION}

Catalytic reduction of $\mathrm{CO}_{2}$ with hydrosilanes as a source of hydrogen has regained attention in recent years. ${ }^{1}$ In contrast to the industry-relevant processes involving utilization of hydrogen as reducing agent, ${ }^{2}$ the application of highly energetic hydrosilanes enables reduction of $\mathrm{CO}_{2}$ under significantly milder conditions due to a thermodynamic stability of the corresponding products 
incorporating $\mathrm{Si}-\mathrm{O}$ bonds. While late-transition metal-based catalysts from various groups $(7-11)^{1,3}$ traditionally play a dominant role in $\mathrm{CO}_{2}$ hydrosilylation, some other examples emerged very recently involving main-group metal organometallics ( $\mathrm{Zn},{ }^{4}$ group 13 ( $\mathrm{Al}$ and $\left.\mathrm{Ga}\right)^{5}$ ) or metal-free systems such as Lewis acids (e.g., $\left.\mathrm{BPh}_{3}\right),{ }^{6}$ Frustrated Lewis Pairs (FLP), ${ }^{7,8}$ phosphazenes, ${ }^{9}$ formate organocatalysts ${ }^{10}$ and abnormal N-heterocyclic carbene. ${ }^{11}$ In addition, both tandem and FLP-like catalytic systems were developed, which are based on binary combinations of a main-group or transition metal precursor and a strong Lewis acid (typically, $\left.\mathrm{B}\left(\mathrm{C}_{6} \mathrm{~F}_{5}\right)_{3}\right): \quad \mathrm{Mg} / \mathrm{B}\left(\mathrm{C}_{6} \mathrm{~F}_{5}\right)_{3},{ }^{12} \mathrm{Zn} / \mathrm{B}\left(\mathrm{C}_{6} \mathrm{~F}_{5}\right)_{3},{ }^{13} \mathrm{Al} / \mathrm{B}\left(\mathrm{C}_{6} \mathrm{~F}_{5}\right)_{3},{ }^{14} \mathrm{Sc} / \mathrm{B}\left(\mathrm{C}_{6} \mathrm{~F}_{5}\right)_{3},{ }^{15} \quad \mathrm{Zr} / \mathrm{B}\left(\mathrm{C}_{6} \mathrm{~F}_{5}\right)_{3},{ }^{16}$ $\mathrm{Re} / \mathrm{B}\left(\mathrm{C}_{6} \mathrm{~F}_{5}\right)_{3}{ }^{17} \mathrm{Ni} / \mathrm{Et}_{3} \mathrm{~B}$ and $\mathrm{Pd}, \mathrm{Pt} / \mathrm{R}_{3} \mathrm{~B}\left(\mathrm{R}=\mathrm{Ph}, \mathrm{C}_{6} \mathrm{~F}_{5}\right) .{ }^{18}$ Depending on the nature of silane, type of catalytic system involved and conditions, the reduction process can be halted at a desired step, thus, allowing to prepare with high selectivity one of the targeted products (Scheme 1) exhibiting a different degree of reduction of carbon atom: silyl formate, , $^{3 \mathrm{a}, \mathrm{b}, \mathrm{d}, \mathrm{e}, \mathrm{g}, \mathrm{h}, \mathrm{i}, \mathrm{k}, \mathrm{m}, \mathrm{m}, \mathrm{cc}, 9 \mathrm{~b}, 10,11,18 \mathrm{~b}}$ bis(silyl)acetal, ${ }^{3 \mathrm{~b}, 6,12,13 b, 17,15 a}$ methoxy-silane $^{3 \mathrm{~m}, 4 \mathrm{~d}, 5 \mathrm{~b}, 9 \mathrm{a}, 13 \mathrm{a}, 15 \mathrm{a}}$ and, finally, methane. ${ }^{\text {3g,5a,7,13,14,15,16,18a }}$

$$
\mathrm{CO}_{2} \underset{\mathrm{R}_{3} \mathrm{SiH}}{\stackrel{\text { [cat] }}{\longrightarrow}} \mathrm{R}_{3} \mathrm{SiO}-\stackrel{\mathrm{O}}{\mathrm{CH}} \underset{\mathrm{R}_{3} \mathrm{SiH}}{\stackrel{\text { [cat] }}{\longrightarrow}}\left(\mathrm{R}_{3} \mathrm{SiO}\right)_{2} \mathrm{CH}_{2} \underset{\mathrm{R}_{3} \mathrm{SiH}}{\stackrel{\text { [cat] }}{\longrightarrow}} \mathrm{R}_{3} \mathrm{SiOCH}_{3} \underset{\mathrm{R}_{3} \mathrm{SiH}}{\stackrel{\text { [cat] }}{\longrightarrow}} \mathrm{CH}_{4}
$$

Scheme 1. Fundamental steps and products of catalytic reduction of $\mathrm{CO}_{2}$ with hydrosilane $\mathrm{R}_{3} \mathrm{SiH}$.

In this work, a new series of homoleptic complexes of group 13 (B, Al, Ga, In) and group 12 elements (Zn) incorporating $N, N$ '-bis(perfluorophenyl)-formamidinate ligands have been prepared and their behavior as catalysts in hydrosilylation of $\mathrm{CO}_{2}$ was studied. We initially hypothesized that during the catalytic process the bidentate LX-type formamidinate ligands ${ }^{19}\{\mathrm{NCN}\}^{-}$in complexes undergo a partial decoordination / rearrangement into an imino-amido form providing a 
N-centered Lewis base function in the close proximity to the vacant Lewis acidic group 13/12 element center, thus giving rise to a FLP form (Scheme 2). ${ }^{20}$ Then, the latter species could presumably trigger the $\mathrm{CO}_{2}$ hydrosilylation mechanism enabling both fixation of $\mathrm{CO}_{2}{ }^{21}$ and activation of hydrosilane ${ }^{22}$ on the two cooperating centers, thus, promoting the formation of silylformate. While discouraging catalytic performance in hydrosilylation of $\mathrm{CO}_{2}$ was initially observed upon utilization of the formamidinate complexes, their binary combinations with $\mathrm{E}_{(}\left(\mathrm{C}_{6} \mathrm{~F}_{5}\right)_{3}$ $(\mathrm{E}=\mathrm{B}, \mathrm{Al})$ appeared to be more efficient catalytic systems. Unexpectedly, very different reactivity patterns were identified for these two-component systems, which are related to the catalyst transformation processes operating under the catalytic conditions.

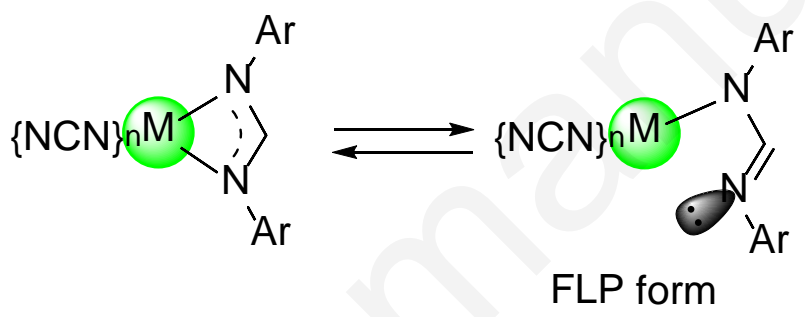

Scheme 2. Formation of a FLP form.

\section{RESULTS AND DISCUSSION}

Synthesis of proligand $N, N^{\prime}$-bis(pentafluorophenyl)methanimidamine (1-H). Introduction of electron-withdrawing groups in ligand platform constitutes a general way to increase electropositivity and, thus, reactivity of active center of a Lewis acid catalyst. Therefore, the use of a poorly donating amidinate-type ligand incorporating $\mathrm{C}_{6} \mathrm{~F}_{5}$ substituents on the electronegative nitrogen atoms was sought in this study. The corresponding proligand 1-H was prepared and isolated in $75 \%$ yield using the published synthetic procedure (Scheme 3). ${ }^{23}$ The molecular structure of $\mathbf{1 - H} \cdot\left(\right.$ benzene) is shown in Figure $\mathrm{S} 1 .^{24}$ 


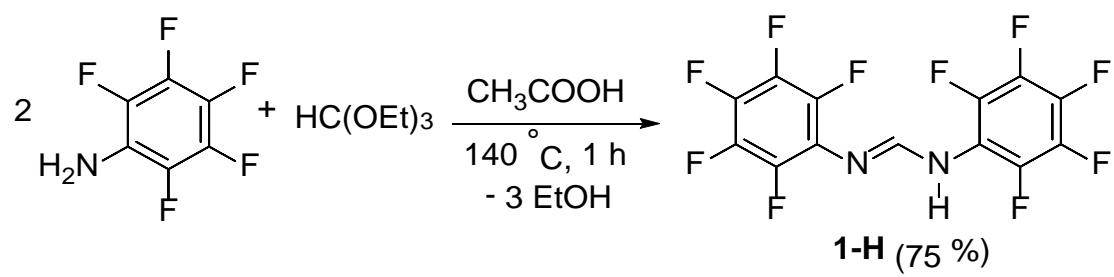

Scheme 3. Synthesis of proligand 1-H.

Synthesis of complexes of B, Al, Ga, In and Zn. Deprotonation of $\mathbf{1 - H}$ with KH in THF resulted in the formation of the corresponding monoanionic potassium salt 1-K (Scheme 4), which was used without isolation in a regular salt-metathesis reaction to prepare the complexes of general formula $\mathrm{M}\{\mathrm{NCN}\}_{3}(\mathrm{M}=\mathrm{B}(\mathbf{1 - B}), \mathrm{Ga}(\mathbf{1 - G a})$ and In (1-In)). On the other hand, complexes 1-Al and 1-Zn were conveniently prepared by one-step alkane elimination reactions between the corresponding metal-alkyl reagent and 1-H proligand (Scheme 5). Compounds 1-B,Zn are soluble in diethyl ether, toluene, benzene, bromobenzene and dichloromethane, while 1-Al,Ga,In exhibit a relatively low solubility in the solvents mentioned above. All complexes are insoluble in aliphatic solvents (heptane and hexane). 1-B appeared to be unstable in THF, rapidly polymerizing this solvent, presumably, by a cationic mechanism. ${ }^{25}$ All the compounds were characterized by NMR spectroscopy, X-ray crystallography and elemental analysis.

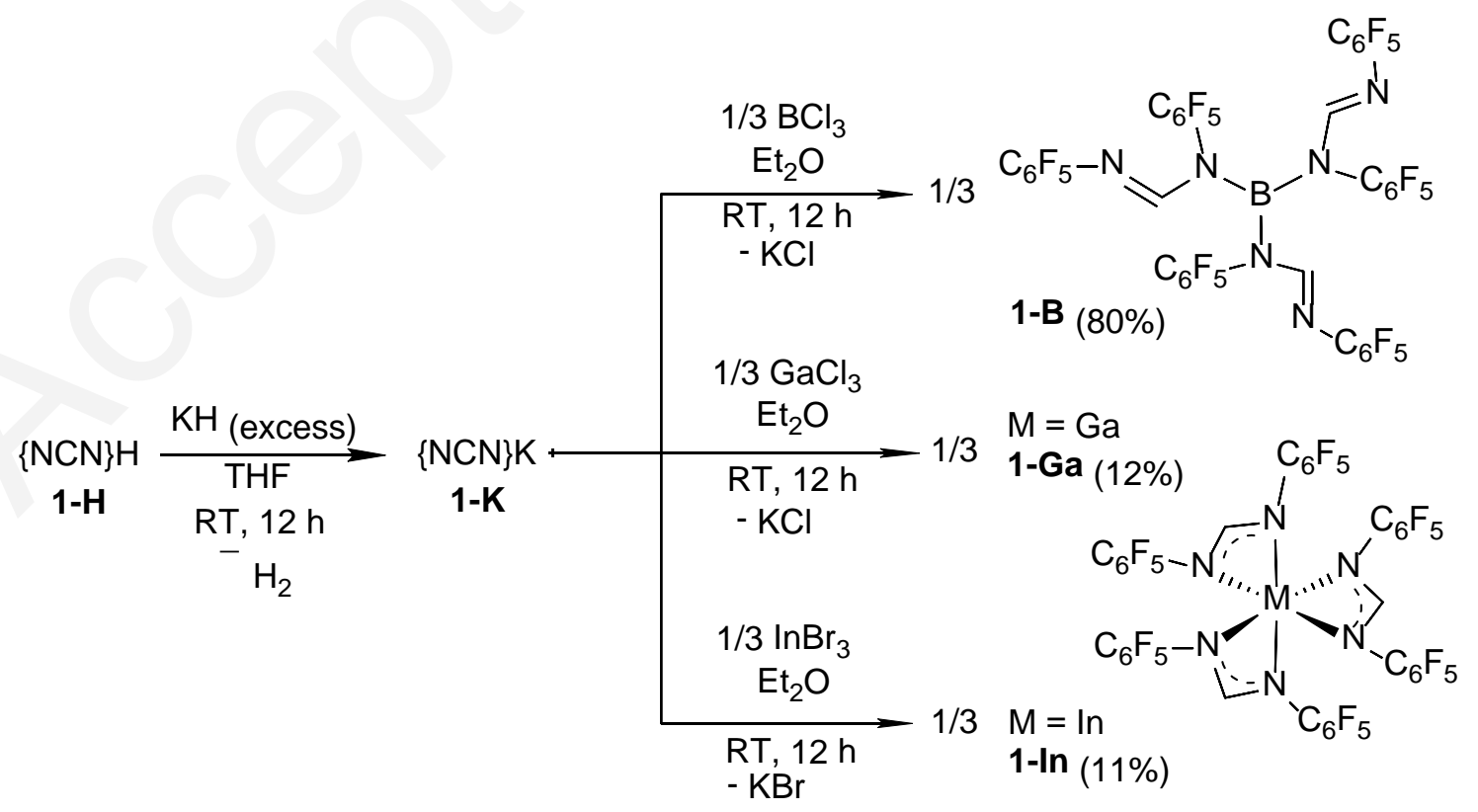


Scheme 4. Synthesis of complexes 1-B, 1-Ga and 1-In by salt metathesis.

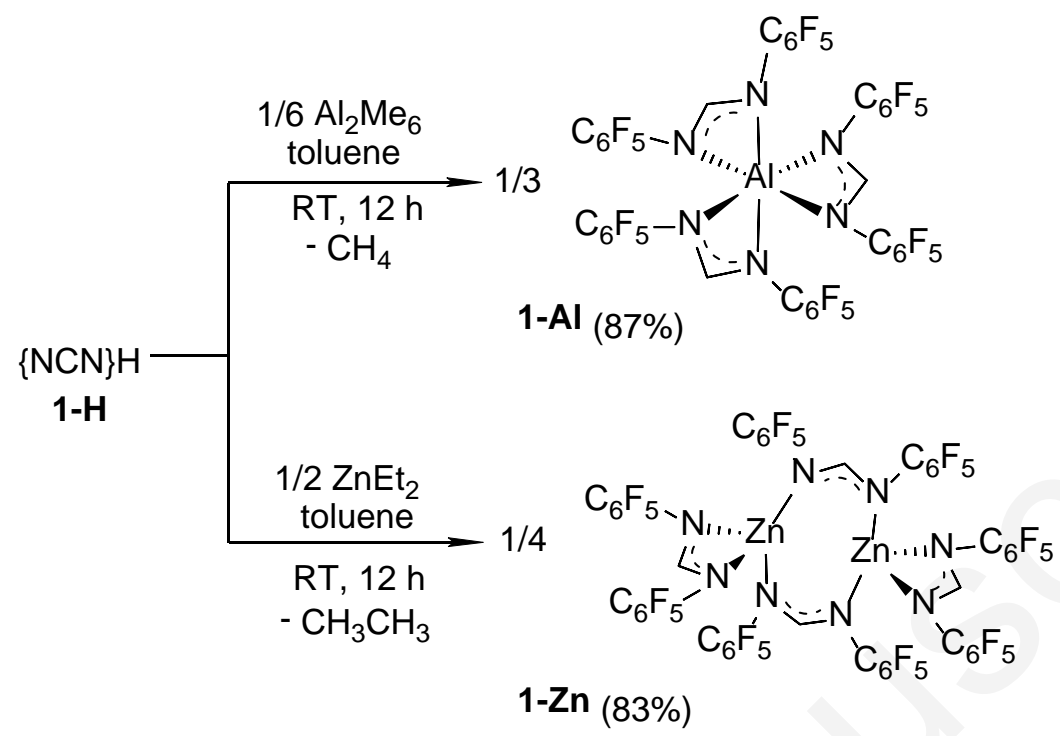

Scheme 5. Synthesis of complexes 1-Al and 1-Zn by $\sigma$-bond metathesis.

Solid-state and solution structures of complexes 1-B,Al,Ga,In,Zn. The solid-state structures of 1-B,Al,Ga,In,Zn complexes were established by X-ray diffraction study (Figures 1-3, Tables 1 and S1).

The crystal cell of 1-B contains two crystallographically independent molecules differing by the reciprocal orientation of the three ligands, thus only one was selected for analysis and discussion. The boron atom in 1-B (Figure 1) features a planar trigonal coordination environment, provided by the equivalent monoanionic imine-formamido ligands each bound with only one nitrogen atom. ${ }^{26}$ Therefore, the coordination of the ligands is different from a regular $\eta^{2}$-mode previously observed in amidinate compounds of type $\{\mathrm{NCN}\} \mathrm{BX}_{2}\left(\mathrm{X}=\mathrm{C}_{6} \mathrm{~F}_{5}{ }^{20 \mathrm{a}, \mathrm{b}}, \mathrm{Cl}^{27}\right)$. Also, the C8-N9 bonds $(1.265(7)-1.273(7) \AA$ ) in the ligand fragments are shortened and the C8-N7 bonds (1.390(8)-1.399(8) $\AA$ ) are elongated as compared to those in the neutral proligand $(1.314(2)$ and 1.315(2) $\AA$, respectively), which is the result of a preferential negative charge localization on the N7 
atom in this formal imine-formamido ligand. The B-N distances in 1-B (1.449(8)-1.462(8) $\AA$ ) are on the longer side of the range observed for those in typical boron-anilido complexes $\mathrm{B}(\mathrm{NMePh})_{3}$ and $\mathrm{B}(\mathrm{NHPh})_{3}(1.428-1.442 \AA) .{ }^{28}$ Also, the molecule exhibits a short F...B contact $(2.915(7) \AA)$ with one ortho-F atom of the closest $\mathrm{C}_{6} \mathrm{~F}_{5}$ aryl ring.

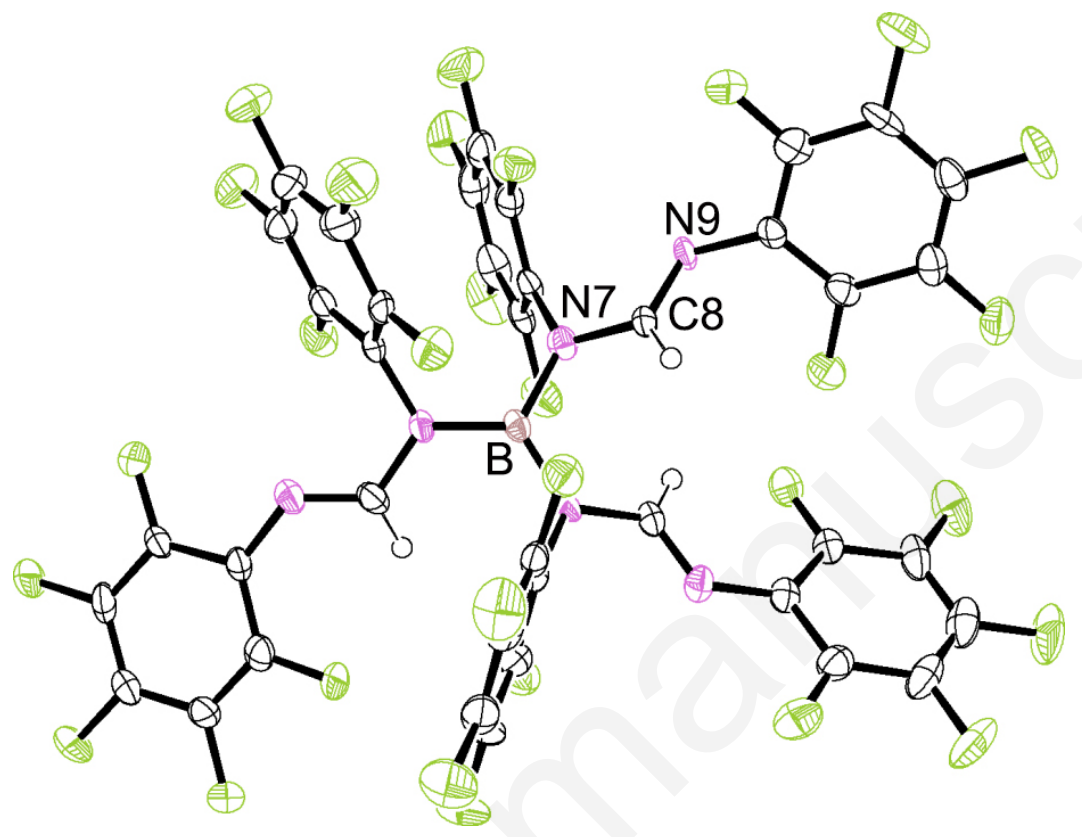

Figure 1. Molecular structure of 1-B (thermal ellipsoids drawn at the 50\% probability).

The X-ray crystallographic study for $\mathbf{1 - A l , ~ G a , ~ I n ~ ( F i g u r e ~ 2 ) ~ r e v e a l e d ~ t h e m ~ t o ~ b e ~ i s o s t r u c t u r a l ~}$ species each featuring the corresponding metal center, chelated by three slightly asymmetrically bound amidinate ligands, in a six-coordinate distorted octahedral environment. Thus, the difference between the corresponding $\mathrm{M}-\mathrm{N}$ bond lengths within the same amidinate ligand does not exceed $0.05 \AA$. Within the series 1-Al, 1-Ga and 1-In, the $\mathrm{M}-\mathrm{N}$ bond distances are elongated, which is in line with the increase of the effective ionic radii of the $\mathrm{M}\left(3^{+}\right)$metal ions. ${ }^{29}$ In $\mathbf{1}-\mathbf{A l}$, the $\mathrm{M}-\mathrm{N}$ bond lengths (1.986(1)-2.021(1) $\AA$ ) are in the normal range of those observed in various amidinate complexes of aluminum (1.884-2.066 $\AA$ ). ${ }^{30,31}$ At the same time, in 1-Ga and 1-In congeners, the corresponding M-N distances (2.022(3)-2.111(3) and 2.214(1)-2.264(1) A, respectively) are on the 
longer side of those reported for their respective analogues (1.996-2.019 and 2.181-2.244 $\AA$, respectively). ${ }^{31}$

The molecular structure of $\mathbf{1 - Z n}$ (Figure 3) consists of a centrosymmetric dimer, in which the two $\left\{\left(\eta^{2}-\mathrm{NCN}\right) \mathrm{Zn}\right\}$ moieties are bound by the two $\mu_{2}$-bridging monoanionic NCN fragments in a "spanned" manner. Thus, each zinc atom has a formal coordination number of 4 and features a distorted tetrahedral environment. The $\mathrm{Zn}-\mathrm{N}$ distances in $\mathbf{1 - Z n}$ are very close to those observed in the relevant complexes incorporating both terminal and bridging $\{\mathrm{NCN}\}^{-}$ligands (2.002-2.092 and 2.000-2.070 Å, respectively). ${ }^{32}$

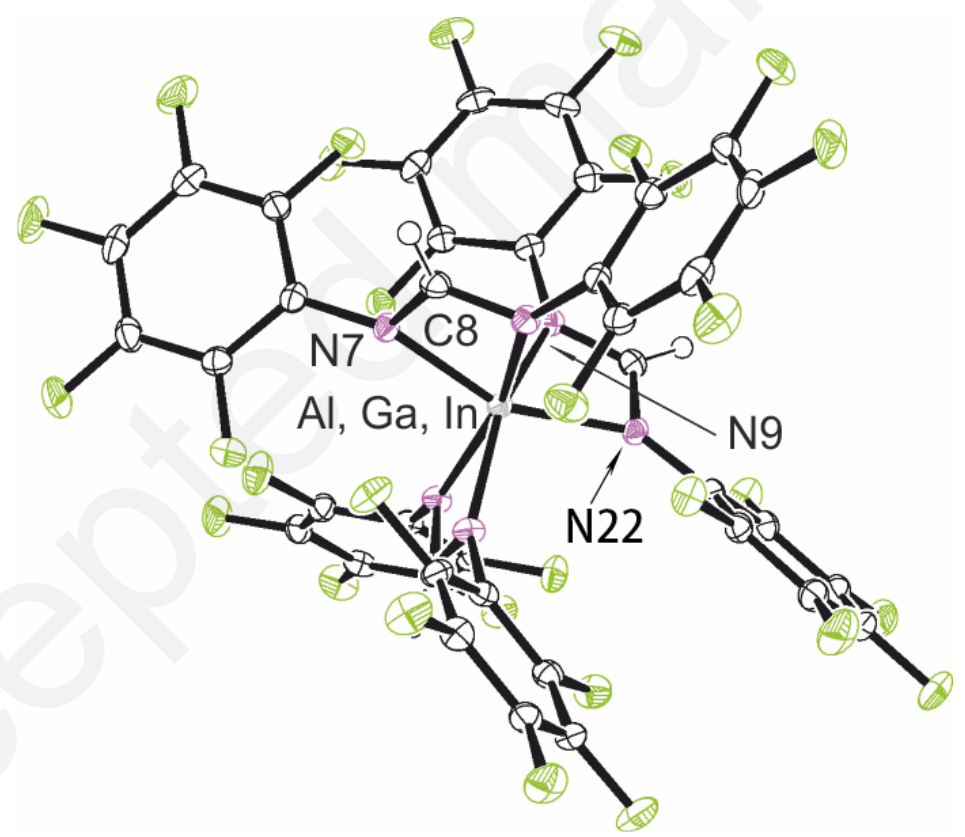

Figure 2. Molecular structure of 1-Al,Ga and 1-In·(benzene) (all solvent molecules are omitted for clarity; thermal ellipsoids drawn at the $50 \%$ probability). 


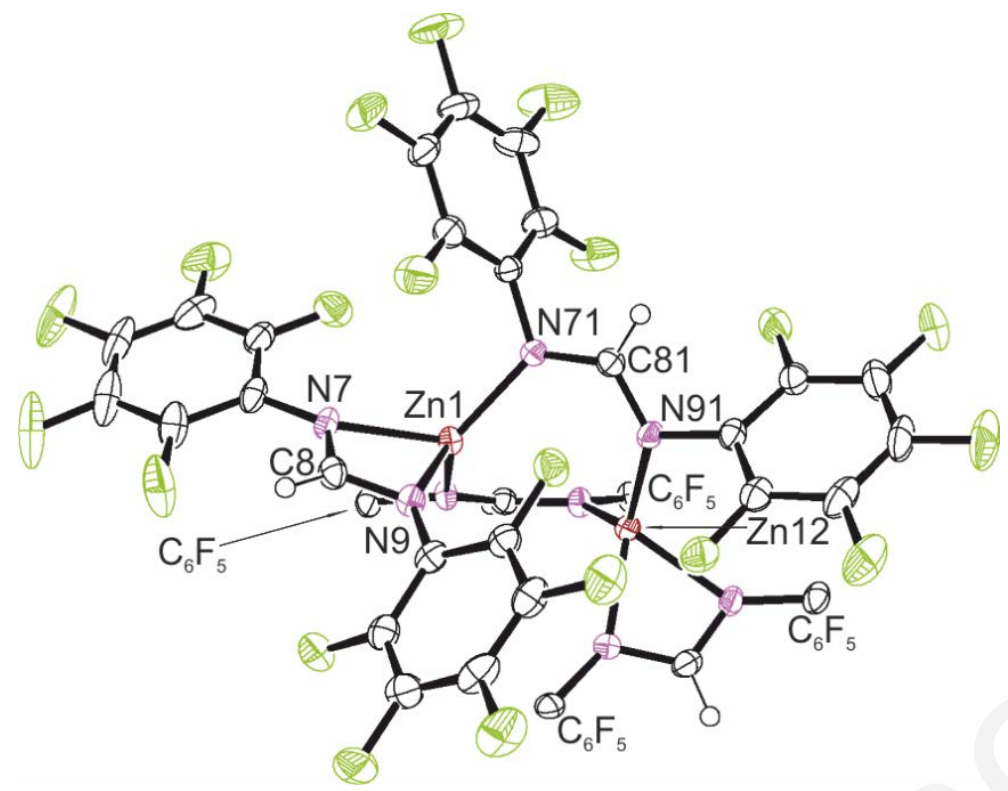

Figure 3. Molecular structure of 1-Zn·(toluene) 2 (all solvent molecules and four $\mathrm{C}_{6} \mathrm{~F}_{5}$ groups are omitted for clarity; thermal ellipsoids drawn at the $50 \%$ probability).

Table 1. Selected bond distances $(\AA)$ and angles $\left(^{\circ}\right)$ for $\mathbf{1 - H , B}, \mathbf{A l}, \mathbf{G a}, \mathbf{I n}, \mathbf{Z n}$.

\begin{tabular}{|c|c|c|c|c|c|c|}
\hline & $1-\mathrm{H}$ & 1-B & 1-Al & 1-Ga & 1-In & $1-\mathrm{Zn}$ \\
\hline \multirow{3}{*}{ M-N7 } & \multirow{3}{*}{-} & $1.444(8)$ & $2.015(1)$ & $2.022(3)$ & $2.214(1)$ & \multirow{3}{*}{$\begin{array}{l}2.050(2) \\
2.050(2)\end{array}$} \\
\hline & & $1.453(8)$ & 2.017(1) & $2.022(3)$ & $2.214(1)$ & \\
\hline & & $1.462(8)$ & $2.021(1)$ & $2.111(3)$ & $2.264(1)$ & \\
\hline \multirow{3}{*}{ M-N9 } & & \multirow{3}{*}{-} & $1.986(1)$ & $2.083(3)$ & $2.260(1)$ & \multirow{3}{*}{$\begin{array}{l}2.069(2) \\
2.069(2)\end{array}$} \\
\hline & - & & $1.996(1)$ & $2.083(3)$ & $2.260(1)$ & \\
\hline & & & $2.002(1)$ & $2.111(3)$ & $2.264(1)$ & \\
\hline \multirow{2}{*}{ M-N71 } & \multirow{2}{*}{-} & \multirow{2}{*}{-} & \multirow{2}{*}{-} & \multirow{2}{*}{ - } & \multirow{2}{*}{-} & $1.977(2)$ \\
\hline & & & & & & $1.977(2)$ \\
\hline \multirow{2}{*}{ M-N91 } & \multirow{2}{*}{-} & \multirow{2}{*}{-} & \multirow{2}{*}{-} & \multirow{2}{*}{-} & \multirow{2}{*}{-} & $1.985(2)$ \\
\hline & & & & & & $1.985(2)$ \\
\hline \multirow{2}{*}{ N71-C78 } & \multirow{2}{*}{-} & \multirow{2}{*}{-} & \multirow{2}{*}{-} & \multirow{2}{*}{-} & \multirow{2}{*}{-} & 1.319(3) \\
\hline & & & & & & $1.319(3)$ \\
\hline N7-C8-N9 & $121.4(2)$ & $120.3(5)$ & $111.7(1)$ & $111.7(3)$ & $114.8(2)$ & $113.00(2)$ \\
\hline N71-C81-N91 & - & - & - & - & - & $124.10(2)$ \\
\hline N7-M-N22 & - & - & $159.4(1)$ & 166.8(3) & $168.8(2)$ & - \\
\hline N7-M-N71 & - & - & - & - & - & $103.8(8)$ \\
\hline
\end{tabular}

${ }^{*} \mathrm{M}=$ metal/element center 
The solution structures of the compounds were also established by NMR spectroscopy. The room-temperature ${ }^{1} \mathrm{H}$ NMR spectra of compounds 1-B,Al,Ga,In and 1-Zn (Figures S2, S7, S10, S12, and $\mathrm{S} 15$, respectively), recorded in $\mathrm{C}_{6} \mathrm{D}_{6}$ or in $\mathrm{C}_{7} \mathrm{D}_{8}$, exhibited in each case a single resonance from the methine $=\mathrm{CH}-$ protons of the formamidinate ligands. Similarly, the corresponding single resonances of the methine carbons were observed in the room-temperature ${ }^{13} \mathrm{C}\left\{{ }^{1} \mathrm{H}\right\}$ NMR spectra of the compounds 1-B,Al, In in $\mathrm{CD}_{2} \mathrm{Cl}_{2}$ and that of $\mathbf{1 - Z n}$ in $\mathrm{C}_{6} \mathrm{D}_{6}$ (Figures S6, S9, S14, and S17, respectively). For an unclear reason, no signals from the complex were observed in the ${ }^{13} \mathrm{C}\left\{{ }^{1} \mathrm{H}\right\}$ NMR spectra at different temperatures of several independently prepared samples of 1-Ga. In the ${ }^{19} \mathrm{~F}\left\{{ }^{1} \mathrm{H}\right\}$ NMR spectra of 1-Al,Ga and 1-In (Figures S8, S11 and S13, respectively), a regular set of narrow signals including one doublet and two triplets from the corresponding $o-\mathrm{F}, p-\mathrm{F}$ and $m-\mathrm{F}$ groups was observed. Therefore, the NMR data obtained for compounds 1-Al,Ga and 1-In are consistent with their solid-state structures in which the three equivalent formamidinate ligands bind metal center in an average symmetrical fashion.

In striking contrast with 1-Al,Ga and 1-In, compounds $\mathbf{1 - B}$ and $\mathbf{1 - Z \mathbf { n } ^ { 3 3 }}$ featured a complex fluxional dynamic behavior in solution in a broad temperature range. For 1-B, the broadened singlet from the methine protons in the ${ }^{1} \mathrm{H}$ NMR in toluene- $d_{8}$ sharpened upon heating up to $80{ }^{\circ} \mathrm{C}$ (Figure 4), whereas upon decreasing the temperature to $-70{ }^{\circ} \mathrm{C}$ the latter signal decoalesced into a series of very broad signals. Also, the three broadened multiplets observed in the ${ }^{19} \mathrm{~F}\left\{{ }^{1} \mathrm{H}\right\} \mathrm{NMR}$ spectrum taken at room temperature, upon cooling down to $-70{ }^{\circ} \mathrm{C}$, split into a complicated series of overlapping multiplets (Figure S4). These observations are indicative of a ligand rearrangement process in the coordination sphere of boron in 1-B and/or of atropoisomerism. Being very slow or frozen at low temperature, both ligand exchange phenomena can afford a mixture of isomers 
incorporating three- or four-coordinated boron centers and featuring different ligand binding motifs and symmetries.

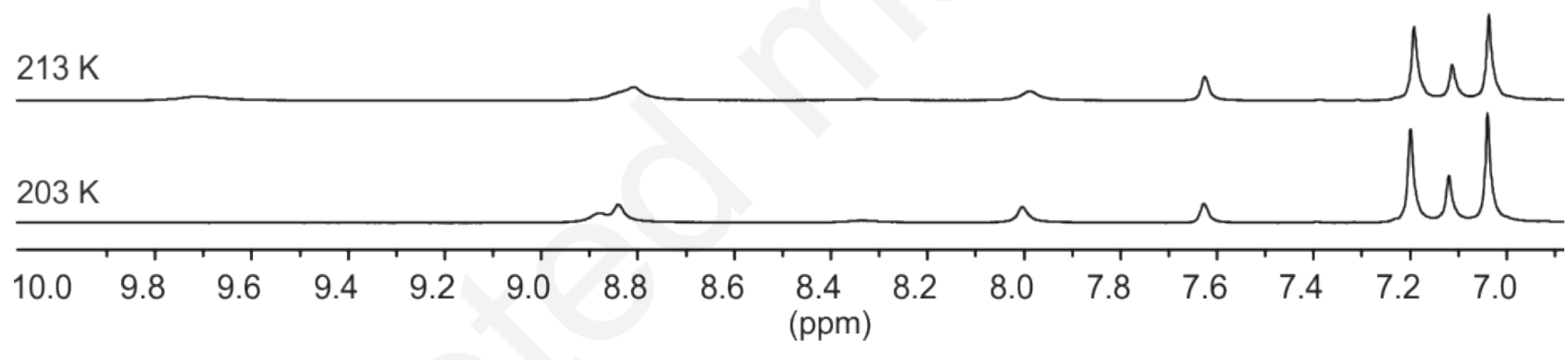

Figure 4. Stack-plot of ${ }^{1} \mathrm{H}$ NMR spectra (400 MHz, toluene- $d_{8}$ ) of 1-B at different temperatures (* stands for residual solvent signals).

A similar process of the ligands rearrangement is apparently operational for the dinuclear $\mathbf{1 - Z n},{ }^{33}$ thus resulting in a rapid exchange at room temperature and the observation of an averagely symmetric species in the corresponding NMR spectra (Figures S15-S17).

Evaluation of Lewis acidity and Acceptor Number (AN). A comparative analysis of the Lewis acidic character of the boron center in 1-B with respect to that of $\mathrm{B}\left(\mathrm{C}_{6} \mathrm{~F}_{5}\right)_{3}$ was performed 
by both theoretical (DFT) and experimental (NMR spectroscopy) techniques (Table 2). In fact, the somewhat higher Lewis acidic character of $\mathbf{1 - B}$ as compared to that of $\mathrm{B}\left(\mathrm{C}_{6} \mathrm{~F}_{5}\right)_{3}$ is in line with the higher positive NBO charge on the boron atom (1.199 vs 0.834 e, respectively). At the same time, the observed higher Lewis acidity of $\mathbf{1 - B}$ cannot be explained by the calculated energy value of the $\pi^{*}$ orbital $\left(\mathrm{E}_{\mathrm{LUMO}}-2.17 \mathrm{eV}\right.$ ), which should have been lower for a more Lewis acidic species. $^{34}$

The relative Lewis acidities of 1-B and $\mathrm{B}\left(\mathrm{C}_{6} \mathrm{~F}_{5}\right)_{3}$ were probed using an experimental Gutmann-Beckett ${ }^{35}$ approach based on the measuring the difference between the ${ }^{31} \mathrm{P}\left\{{ }^{1} \mathrm{H}\right\}$ NMR chemical shift of the free $\mathrm{Et}_{3} \mathrm{P}=\mathrm{O}(\delta$ 45.7) and that of the corresponding Lewis acid adduct. The 1-B/Et $3 \mathrm{P}=\mathrm{O}$ system showed a larger $\Delta \delta\left({ }^{31} \mathrm{P}\right)$ difference $(33.0 \mathrm{ppm})$ in comparison to the $\mathrm{B}\left(\mathrm{C}_{6} \mathrm{~F}_{5}\right)_{3} / \mathrm{Et}_{3} \mathrm{P}=\mathrm{O}$ reference $(\Delta \delta 30.0 \mathrm{ppm})$, thus corroborating a higher Lewis acidity of the boron center in 1-B. In terms of AN, the Lewis acidity of 1-B is comparable with that of catechol ester $\mathrm{B}\left(\mathrm{C}_{6} \mathrm{~F}_{5}\right)\left(\mathrm{O}_{2} \mathrm{C}_{6} \mathrm{H}_{4}\right)(\mathrm{AN}=81.5)$ and that of $\mathrm{HB}\left(2,4,6-\left(\mathrm{CF}_{3}\right)_{3} \mathrm{C}_{6} \mathrm{H}_{2}\right)(\mathrm{AN}=83.3)$, and is lower than that of $\mathrm{B}\left(\mathrm{C}_{6} \mathrm{~F}_{5}\right)_{2}\left(\mathrm{OC}_{6} \mathrm{~F}_{5}\right)(\mathrm{AN}=86.2)$ or $\mathrm{B}\left(\mathrm{C}_{6} \mathrm{~F}_{5}\right)\left(\mathrm{OC}_{6} \mathrm{~F}_{5}\right)_{2}(\mathrm{AN}=87.3) .{ }^{36,37,38}$

Measurements of the differences in chemical shifts $\left(\Delta \delta\left({ }^{31} \mathrm{P}\right)\right)$ upon reaction between $\mathrm{Et}_{3} \mathrm{P}=\mathrm{O}$ and 1-M (M = Al, Ga, In, Zn), carried out under identical conditions, afforded the following relative order of the Lewis acidities: 1-Al $<\mathbf{1 - Z n}<\mathbf{1}-\mathbf{I n}<\mathrm{B}\left(\mathrm{C}_{6} \mathrm{~F}_{5}\right)_{3}<\mathbf{1 - B} \leq \mathbf{1 - G a}$. Unexpectedly, the experimentally determined order of the Lewis acidities of 1-M ( $\mathbf{M}=\mathrm{Al}, \mathrm{Ga}, \mathrm{In}, \mathrm{Zn})$ stays in variance with the trend of computed NBO charges. For instance, the softer within the whole series Lewis acid 1-Al exhibited the greater NBO charge on the aluminum center (1.897 e).

Table 2. DFT calculated HOMO-LUMO gaps, dipole moments and NBO charges, and experimentally measured $\Delta \delta\left({ }^{31} \mathrm{P}\right)$ and $\mathrm{AN}$ for $\mathrm{B}\left(\mathrm{C}_{6} \mathrm{~F}_{5}\right)_{3}, \mathbf{1 - B}, \mathbf{1 - A l}, \mathbf{1 - G a}, \mathbf{1 - I n}$ and 1-Zn. ${ }^{a}$ 


\begin{tabular}{lcccccc}
\hline Complex & $\mathbf{B}\left(\mathbf{C}_{\mathbf{6}} \mathbf{F}_{5}\right)_{\mathbf{3}}$ & $\mathbf{1 - B}$ & $\mathbf{1 - A l}$ & $\mathbf{1 - G a}$ & $\mathbf{1 - I n}$ & $\mathbf{1 - Z n}$ \\
\hline E HOMO, [eV] & -7.08 & -6.31 & -7.26 & -6.19 & - & -5.99 \\
ELUMO, [eV] $_{\text {HOMO-LUMO gap, [eV] }}$ & -2.97 & -2.17 & -3.92 & -1.59 & - & -1.51 \\
Hipole moment, [D] & 4.11 & 4.14 & 3.34 & 4.60 & - & 4.48 \\
NBO charge on M, [e] & 0.03 & 3.24 & 0.18 & 0.23 & - & 1.07 \\
& 0.834 & 1.199 & 1.897 & 1.694 & - & 1.303 \\
$\Delta \delta\left({ }^{31} \mathrm{P}\right),[\mathrm{ppm}]^{b}$ & & & & & & \\
$\mathrm{AN}^{d}$ & $30.0^{c}$ & 33.0 & 4.7 & 33.4 & 23.6 & 17.7 \\
\hline
\end{tabular}

${ }^{a}$ B3PW91/6-31G(d,p), SMD (toluene); ${ }^{b}$ the difference between the ${ }^{31} \mathrm{P}\left\{{ }^{1} \mathrm{H}\right\}$ NMR chemical shifts of the free $\mathrm{Et}_{3} \mathrm{P}=\mathrm{O}$ and that of its adduct with the substrate measured in benzene- $d_{6}$ at room temperature; ${ }^{c}$ the reported value in $\mathrm{C}_{6} \mathrm{D}_{6}$ at room temperature, $30.2 \mathrm{ppm} ;{ }^{37}{ }^{d}$ calculated using the following expression $\mathrm{AN}=2.21\left(\delta{ }^{31} \mathrm{P}_{\mathrm{LA} \cdot \mathrm{Et} 3 \mathrm{PO}}-41\right) .{ }^{38}$

Catalytic $\mathrm{CO}_{2}$ hydrosilylation. Hydrosilylation of $\mathrm{CO}_{2}$ with $\mathrm{Et}_{3} \mathrm{SiH}$ in the presence of 1-B,Al,Ga,In and 1-Zn was investigated. Strong Lewis acids, namely $B\left(\mathrm{C}_{6} \mathrm{~F}_{5}\right)_{3}$ and $\mathrm{Al}\left(\mathrm{C}_{6} \mathrm{~F}_{5}\right)_{3}$ (toluene $)_{0.5}$, were studied as catalysts for benchmarking purposes, and then, as cocatalysts $^{14}$ in a tandem combination with 1-B,Al,Ga,In and Zn. For the most performing catalytic systems, several parameters were examined such as the pressure of $\mathrm{CO}_{2}\left(1 \mathrm{bar}\left(n\left(\mathrm{CO}_{2}\right)_{0}\right.\right.$ $\left./ n(\mathrm{Si}-\mathrm{H})_{0}=1: 1\right)$ and 6 bar $\left.\left(n\left(\mathrm{CO}_{2}\right)_{0} / n(\mathrm{Si}-\mathrm{H})_{0}=6: 1\right)\right),{ }^{39}$ temperature and reaction time, the nature of solvent (benzene vs bromobenzene) and the concentration of pre-catalyst. In addition, hydrosilylation using different hydrosilanes $\left(\mathrm{PhSiMe}_{2} \mathrm{H}, \mathrm{Ph}_{2} \mathrm{SiH}_{2}, \mathrm{PhSiH}_{3}, \mathrm{Ph}_{3} \mathrm{SiH}\right.$ and $\left.(\mathrm{EtO})_{3} \mathrm{SiH}\right)$ was also explored. The reactions were monitored by ${ }^{1} \mathrm{H}$ NMR spectroscopy detecting the formation of the four consecutive products (Scheme 6, a-d). ${ }^{40}$ Formation of disiloxane $\mathrm{Et}_{3} \mathrm{SiOSiEt}_{3}(\mathbf{e})$, the second product of the two last reduction steps, was corroborated by GC-MS analysis.

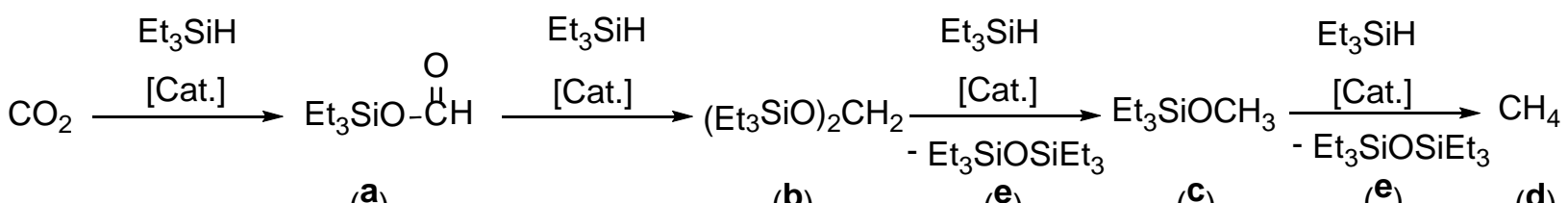

$(\mathbf{e})$

$\left(c^{c}\right)$

$\left({ }^{e}\right)$ 
Scheme 6. Products of the catalytic reduction of $\mathrm{CO}_{2}$ with $\mathrm{Et}_{3} \mathrm{SiH}$.

In accordance with previous reports, ${ }^{14}$ the benchmarking reduction reaction of $\mathrm{CO}_{2}$ with $\mathrm{Et}_{3} \mathrm{SiH}$ under our experimental conditions $\left([\mathrm{B}]_{0}=[\mathrm{Al}]_{0}=5 \mathrm{~mol} \%, \mathrm{C}_{6} \mathrm{D}_{5} \mathrm{Br}, 25\right.$ or $80{ }^{\circ} \mathrm{C}$, 1 or 6 bar $\mathrm{CO}_{2}$ ) was not productive with $\mathrm{B}\left(\mathrm{C}_{6} \mathrm{~F}_{5}\right)_{3}$ (Table S2, entries 1-3), and the same reaction with $\mathrm{Al}\left(\mathrm{C}_{6} \mathrm{~F}_{5}\right)_{3}(\text { toluene })_{0.5}$ (entry 4$)$ resulted in a poor conversion of hydrosilane $(16 \%)$ towards a $(<1 \%)$ and $\mathbf{b}(15 \%)$. Upon using the tandem system $\mathrm{B}\left(\mathrm{C}_{6} \mathrm{~F}_{5}\right)_{3} / \mathrm{Al}\left(\mathrm{C}_{6} \mathrm{~F}_{5}\right)_{3}$ (toluene $)_{0.5}{ }^{14}$ (entries 5-7), the reduction appeared to be somewhat slower under our experimental conditions, and high hydrosilane conversions and methane yields ( $>80$ and $>70 \%$, respectively) were achieved at 6 bar $\mathrm{CO}_{2}$ pressure after 48 hours (entry 7).

All single-component systems 1-M appeared to be either completely inactive ( $M=\mathrm{Ga}$, In, Zn) or only sluggishly active $(\mathrm{M}=\mathrm{B}, \mathrm{Al})$ in the reduction of $\mathrm{CO}_{2}\left(1\right.$ bar) with $\mathrm{Et}_{3} \mathrm{SiH}$, attempted both in $\mathrm{C}_{7} \mathrm{D}_{8}$ and in $\mathrm{C}_{6} \mathrm{D}_{5} \mathrm{Br}$ (Table 3, entries 1-3; Table 4, entry 1; Table S3, entries 1, 6 and 10). Therefore, their two-component combinations with $\mathrm{B}\left(\mathrm{C}_{6} \mathrm{~F}_{5}\right)_{3}$ or $\mathrm{Al}\left(\mathrm{C}_{6} \mathrm{~F}_{5}\right)_{3}$ (toluene $)_{0.5}$ were systematically studied as precatalysts (Tables 3,4 and S3). With binary system $\mathbf{1 - B} / \mathrm{B}\left(\mathrm{C}_{6} \mathrm{~F}_{5}\right)_{3}$ in $\mathrm{C}_{6} \mathrm{D}_{5} \mathrm{Br}$, the three products were observed by ${ }^{1} \mathrm{H}$ NMR spectroscopy (entries $4-7$, Table 3$)^{40}$ are: major $\left(\mathrm{Et}_{3} \mathrm{SiO}\right)_{2} \mathrm{CH}_{2}$ (b) and methane (d), and minor $\mathrm{Et}_{3} \mathrm{SiOCH}_{3}$ (c); no formation of the primary reduction product, silylformate $\mathrm{Et}_{3} \mathrm{SiOC}(\mathrm{O}) \mathrm{H}(\mathbf{a})$, was observed in all cases, regardless the conditions. On the other hand, with 1-B/Al $\left(\mathrm{C}_{6} \mathrm{~F}_{5}\right)_{3}$ (toluene $)_{0.5}$ congener, only products $\mathbf{b}$ and $\mathbf{d}$ were detected. ${ }^{41}$ These results are not surprising, and in the previous studies using various catalytic systems have been explained by an extremely high activity of a towards reduction with $\mathrm{Et}_{3} \mathrm{SiH}^{3 \mathrm{ff}, 6,16}$

Comparable activities were found for $\mathbf{1 - B} / \mathrm{B}\left(\mathrm{C}_{6} \mathrm{~F}_{5}\right)_{3}$ and $\mathbf{1 - B} / \mathrm{Al}\left(\mathrm{C}_{6} \mathrm{~F}_{5}\right)_{3}$ (toluene $)_{0.5}$ at $80{ }^{\circ} \mathrm{C}$ both at 1 bar and 6 bar $\mathrm{CO}_{2}$ pressure (compare entries 5/6 vs 8/10, respectively). At the same time, 
a complete hydrosilane conversion was achieved with $1-B / B\left(C_{6} F_{5}\right)_{3}$, at least, two times faster than that with 1-B/ $\mathrm{Al}\left(\mathrm{C}_{6} \mathrm{~F}_{5}\right)_{3}$ (toluene) $)_{0.5}$ under the same conditions (compare entries 7 and 11).

The attempt to perform the reduction reaction under conditions potentially generating a FLP $\mathrm{P}^{21 \mathrm{~b}, 42}$ upon using an equimolar mixture of $\mathbf{1 - B}$ and $\mathrm{tBu}_{3} \mathrm{P}$ failed (entry 12). No conversion of $\mathrm{Et}_{3} \mathrm{SiH}$ was observed in this case, and no products were detected. 
Table 3. Catalytic reduction of $\mathrm{CO}_{2}$ with $\mathrm{Et}_{3} \mathrm{SiH}$ in the presence of 1-B. ${ }^{[\mathrm{a}]}$

\begin{tabular}{|c|c|c|c|c|c|c|c|c|c|c|c|}
\hline \multirow[t]{2}{*}{ Entry } & \multirow{2}{*}{$\begin{array}{c}\text { Precat } \\
{[\mathrm{mol} \%]}\end{array}$} & \multirow{2}{*}{ 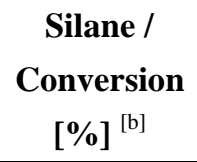 } & \multirow[t]{2}{*}{ Solvent } & \multirow{2}{*}{$\begin{array}{l}\mathbf{P}_{\mathrm{CO} 2} \\
\text { [bar] }\end{array}$} & \multirow{2}{*}{$\begin{array}{c}\mathbf{T} \\
{\left[{ }^{\circ} \mathbf{C}\right]}\end{array}$} & \multirow{2}{*}{$\begin{array}{c}t \\
{[h]}\end{array}$} & \multicolumn{5}{|c|}{$\begin{array}{c}\text { Si products / } \\
\text { Conversion [\%] }{ }^{[\mathrm{c}]}\end{array}$} \\
\hline & & & & & & & $\mathbf{a}$ & $\mathbf{b}$ & c & d & $\mathbf{f}+\mathbf{g}$ \\
\hline $1^{[\mathrm{d}]}$ & $\begin{array}{l}\text { 1-B } \\
(0.5)\end{array}$ & $\begin{array}{c}\mathrm{Et}_{3} \mathrm{Si}-\mathrm{H} \\
0\end{array}$ & $\mathrm{C}_{7} \mathrm{D}_{8}$ & 1 & 25 & 48 & 0 & 0 & 0 & 0 & 0 \\
\hline $2^{[\mathrm{d}]}$ & $\begin{array}{l}\text { 1-B } \\
(0.5)\end{array}$ & $\begin{array}{c}\mathrm{Et}_{3} \mathrm{Si}-\mathrm{H} \\
0\end{array}$ & $\mathrm{C}_{7} \mathrm{D}_{8}$ & 1 & 80 & 16 & 0 & 0 & 0 & 0 & 0 \\
\hline 3 & $\begin{array}{l}\text { 1-B } \\
(10)\end{array}$ & $\begin{array}{c}\mathrm{Et}_{3} \mathrm{Si}-\mathrm{H} \\
6\end{array}$ & $\mathrm{C}_{6} \mathrm{D}_{5} \mathrm{Br}$ & 1 & 80 & 48 & 0 & 5.1 & 0 & 0 & 0 \\
\hline 4 & $\begin{array}{c}\text { 1-B } \\
(5.0) \\
\mathrm{B}\left(\mathrm{C}_{6} \mathrm{~F}_{5}\right)_{3} \\
(5.0)\end{array}$ & $\begin{array}{c}\mathrm{Et}_{3} \mathrm{Si}-\mathrm{H} \\
18\end{array}$ & $\mathrm{C}_{6} \mathrm{D}_{5} \mathrm{Br}$ & 1 & 80 & 5 & 0 & 1.5 & 0.5 & 2.1 & 8.3 \\
\hline 5 & $\begin{array}{c}\text { 1-B } \\
(5.0) \\
\mathrm{B}\left(\mathrm{C}_{6} \mathrm{~F}_{5}\right)_{3} \\
(5.0)\end{array}$ & $\begin{array}{c}\mathrm{Et}_{3} \mathrm{Si}-\mathrm{H} \\
36\end{array}$ & $\mathrm{C}_{6} \mathrm{D}_{5} \mathrm{Br}$ & 1 & 80 & 48 & 0 & 11.0 & 0.6 & 8.1 & 9.1 \\
\hline $6^{[\mathrm{e}]}$ & $\begin{array}{c}\text { 1-B } \\
(5.0) \\
\mathrm{B}\left(\mathrm{C}_{6} \mathrm{~F}_{5}\right)_{3} \\
(5.0)\end{array}$ & $\begin{array}{c}\mathrm{Et}_{3} \mathrm{Si}-\mathrm{H} \\
46\end{array}$ & $\mathrm{C}_{6} \mathrm{D}_{5} \mathrm{Br}$ & 6 & 80 & 5 & 0 & 7.9 & 0.7 & 22.5 & 8.8 \\
\hline $7^{[\mathrm{e}]}$ & $\begin{array}{c}\text { 1-B } \\
(5.0) \\
\mathrm{B}\left(\mathrm{C}_{6} \mathrm{~F}_{5}\right)_{3} \\
(5.0)\end{array}$ & $\begin{array}{c}\mathrm{Et}_{3} \mathrm{Si}-\mathrm{H} \\
>98\end{array}$ & $\mathrm{C}_{6} \mathrm{D}_{5} \mathrm{Br}$ & 6 & 80 & 25 & 0 & 12.1 & 1.1 & 69.1 & 10.7 \\
\hline
\end{tabular}




\begin{tabular}{|c|c|c|c|c|c|c|c|c|c|c|c|}
\hline 8 & $\begin{array}{c}\text { 1-B } \\
(5.0) \\
\mathrm{Al}\left(\mathrm{C}_{6} \mathrm{~F}_{5}\right)_{3}(\text { toluene })_{0.5} \\
(5.0) \\
\end{array}$ & $\begin{array}{c}\mathrm{Et}_{3} \mathrm{Si}-\mathrm{H} \\
18\end{array}$ & $\mathrm{C}_{6} \mathrm{D}_{5} \mathrm{Br}$ & 1 & 80 & 5 & 0 & 2.3 & 0 & 0.8 & 6.0 \\
\hline 9 & $\begin{array}{c}\text { 1-B } \\
(5.0) \\
\mathrm{Al}\left(\mathrm{C}_{6} \mathrm{~F}_{5}\right)_{3}(\text { toluene })_{0.5} \\
(5.0) \\
\end{array}$ & $\begin{array}{c}\mathrm{Et}_{3} \mathrm{Si}-\mathrm{H} \\
38\end{array}$ & $\mathrm{C}_{6} \mathrm{D}_{5} \mathrm{Br}$ & 1 & 80 & 48 & 0 & 5.9 & 0 & 11.4 & 8.5 \\
\hline $10^{[\mathrm{e}]}$ & $\begin{array}{c}\text { 1-B } \\
(5.0) \\
\mathrm{Al}\left(\mathrm{C}_{6} \mathrm{~F}_{5}\right)_{3}(\text { toluene })_{0.5} \\
(5.0) \\
\end{array}$ & $\begin{array}{c}\mathrm{Et}_{3} \mathrm{Si}-\mathrm{H} \\
38\end{array}$ & $\mathrm{C}_{6} \mathrm{D}_{5} \mathrm{Br}$ & 6 & 80 & 5 & 0 & 17.8 & 0 & 1.3 & 7.4 \\
\hline $11^{[\mathrm{e}]}$ & $\begin{array}{c}\text { 1-B } \\
(5.0) \\
\mathrm{Al}\left(\mathrm{C}_{6} \mathrm{~F}_{5}\right)_{3}(\text { toluene })_{0.5} \\
(5.0) \\
\end{array}$ & $\begin{array}{c}\mathrm{Et}_{3} \mathrm{Si}-\mathrm{H} \\
95\end{array}$ & $\mathrm{C}_{6} \mathrm{D}_{5} \mathrm{Br}$ & 6 & 80 & 48 & 0 & 17.7 & 0 & 54.2 & 9.3 \\
\hline $12^{[\mathrm{e}]}$ & $\begin{array}{l}\mathbf{1 - B} \\
(10.0) \\
\mathrm{tBu}_{3} \mathrm{P} \\
(10.0)\end{array}$ & $\begin{array}{c}\mathrm{Et}_{3} \mathrm{Si}-\mathrm{H} \\
0\end{array}$ & $\mathrm{C}_{6} \mathrm{D}_{5} \mathrm{Br}$ & 6 & 80 & 48 & 0 & 0 & 0 & 0 & 0 \\
\hline
\end{tabular}

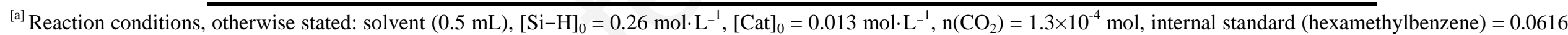

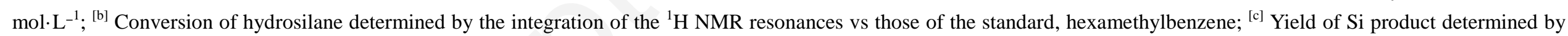

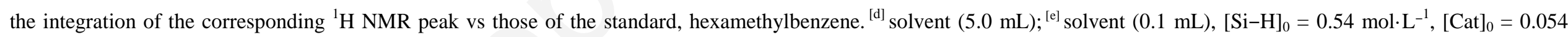
$\mathrm{mol} \cdot \mathrm{L}^{-1}, n\left(\mathrm{CO}_{2}\right)_{0}=3.2 \times 10^{-4} \mathrm{~mol}$, internal standard (hexamethylbenzene) $=0.0615 \mathrm{~mol} \cdot \mathrm{L}^{-1}$; 
Astonishingly, a binary system 1-Al/B $\left(\mathrm{C}_{6} \mathrm{~F}_{5}\right)_{3}$ appeared to be the most active within the whole series of combinations (Table 4). With this system, a complete hydrosilane conversion can be achieved within 5 to $10 \mathrm{~h}$ at $80{ }^{\circ} \mathrm{C}$ both at 1 and 6 bar $\mathrm{CO}_{2}$ pressure (entries 2-4). By analogy with 1-B/B $\left(\mathrm{C}_{6} \mathrm{~F}_{5}\right)_{3}$, the same three products were detected by ${ }^{1} \mathrm{H}$ NMR spectroscopy: major $\mathbf{b}$ and $\mathbf{d}$, and minor c. Congener 1-Al/Al $\left(\mathrm{C}_{6} \mathrm{~F}_{5}\right)_{3}$ (toluene $)_{0.5}$ (entries 16-19) provided a low conversion ( $\left.<25 \%\right)$ of $\mathrm{Et}_{3} \mathrm{SiH}$, which could be achieved at $80{ }^{\circ} \mathrm{C}$ and at 6 bar only after $48 \mathrm{~h}$ affording small amounts of $\mathbf{a}-\mathbf{c}$ and traces of $\mathbf{d}$.

Therefore, the mixed catalytic system 1-Al/B( $\left.\mathrm{C}_{6} \mathrm{~F}_{5}\right)_{3}$ was successfully applied using lower loading $\left(\left[\mathrm{Al}_{0}=[\mathrm{B}]_{0}=1 \mathrm{~mol} \%\right)\right.$. Though, moderate conversion of hydrosilane was obtained at room temperature and 1 bar $\mathrm{CO}_{2}$ pressure in $\mathrm{C}_{6} \mathrm{D}_{5} \mathrm{Br}$ or in $\mathrm{C}_{6} \mathrm{D}_{6}$ (entries 5 and 6 , respectively), at higher pressure (6 bar; entries 7 and 8, respectively; Figure S22), $\mathrm{Et}_{3} \mathrm{SiH}$ was completely consumed within a shorter period of time $(30 \mathrm{~h})$. At higher temperature $\left(80^{\circ} \mathrm{C}\right)$ and 6 bar $\mathrm{CO}_{2}$ pressure, the complete consumption of hydrosilane can be achieved within $5 \mathrm{~h}$ in $\mathrm{C}_{6} \mathrm{D}_{5} \mathrm{Br}$ and within $10 \mathrm{~h}$ in $\mathrm{C}_{6} \mathrm{D}_{6}$ (entries 9 and 11, respectively). In order to probe the critical role of $\mathbf{1}$-Al as cocatalyst, a series of experiments with different respective cocatalyst ratios was conducted (entries 12-15). Thus, upon using a larger proportion of 1-Al $\left([\mathbf{1}-\mathbf{A l}]_{0} /\left[\mathrm{B}\left(\mathrm{C}_{6} \mathrm{~F}_{5}\right)_{3}\right]_{0}=3: 1\right.$, entries 13 and 15), the high silane conversion was achieved faster and the yield of $\mathbf{b}$ was systematically higher. This argues in favor of predominant contribution of $\mathbf{1 - A l}$ in the generation of catalytically active species responsible for the conversion of $\mathrm{Et}_{3} \mathrm{SiH}$ to $\mathbf{b}$.

Under the optimized conditions ([1-Al $]_{0} /\left[\mathrm{B}\left(\mathrm{C}_{6} \mathrm{~F}_{5}\right)_{3}\right]_{0}=1$ mol\%, $\mathrm{C}_{6} \mathrm{D}_{6}, 6$ bar, $\left.80{ }^{\circ} \mathrm{C}\right)$, hydrosilylation of $\mathrm{CO}_{2}$ with various hydrosilanes was then studied (Table 5). The composition of each crude reaction mixture was determined by ${ }^{1} \mathrm{H}$ NMR spectroscopy. Thus, the following 
hydrosilane reactivity trend was revealed: $\mathrm{PhMe}_{2} \mathrm{SiH}>\mathrm{Et}_{3} \mathrm{SiH}>\mathrm{PhSiH}_{3}>\mathrm{Ph}_{2} \mathrm{SiH}_{2}>\mathrm{Ph}_{3} \mathrm{SiH}>$ $(\mathrm{EtO})_{3} \mathrm{SiH}$. The observed trend is only partially in agreement with both thermodynamic hydricity $\left(\mathrm{Ph}_{3} \mathrm{SiH}=\mathrm{PhMe}_{2} \mathrm{SiH}>\mathrm{Et}_{3} \mathrm{SiH}>\mathrm{Ph}_{2} \mathrm{SiH}_{2}>\mathrm{PhSiH}_{3}\right)^{43}$ and kinetic nucleophilicity $\left(\mathrm{PhMe}_{2} \mathrm{SiH}>\right.$ $\left.\mathrm{Et}_{3} \mathrm{SiH}>\mathrm{Ph}_{3} \mathrm{SiH}>\mathrm{Ph}_{2} \mathrm{SiH}_{2}>\mathrm{PhSiH}_{3}>(\mathrm{EtO})_{3} \mathrm{SiH}\right)^{44}$ tendencies, and is inconsistent with those for the series of relatively bulkier hydrosilanes $\mathrm{Ph}_{3} \mathrm{SiH}, \mathrm{Ph}_{2} \mathrm{SiH}_{2}$ and $\mathrm{PhSiH}_{3}$ demonstrating quite the opposite corresponding trends. Given the observed trend can be only in part rationalized in terms of the thermodynamic and kinetic stability of the corresponding $\mathrm{R}_{3} \mathrm{SiH}$...LA adducts $(\mathrm{LA}=$ Lewis Acid, e.g. $\left.\mathrm{B}\left(\mathrm{C}_{6} \mathrm{~F}_{5}\right)_{3}\right)$ responsible for the hydride transfer, ${ }^{14,15}$ we surmised that the steric hindrance of hydrosilanes also contributes to the stability of the titled key adducts and, thus, to the global activity.

The reactions with $\mathrm{PhMe}_{2} \mathrm{SiH}$ and $\mathrm{PhSiH}_{3}$ afforded methane (d) as the major product, while in the presence of $\mathrm{Ph}_{2} \mathrm{SiH}_{2}$, mostly b was obtained. 
Table 4. Catalytic reduction of $\mathrm{CO}_{2}$ with $\mathrm{Et}_{3} \mathrm{SiH}$ in the presence of 1-Al. ${ }^{[\mathrm{a}]}$

\begin{tabular}{|c|c|c|c|c|c|c|c|c|c|c|c|}
\hline \multirow[t]{2}{*}{ Entry } & \multirow{2}{*}{$\begin{array}{c}\text { Precat } \\
\text { [mol\%] }\end{array}$} & \multirow{2}{*}{$\begin{array}{c}\text { Silane / } \\
\text { Conversion } \\
\text { [\%] }^{[\mathrm{b}]} \\
\end{array}$} & \multirow[t]{2}{*}{ Solvent } & \multirow{2}{*}{$\begin{array}{c}\mathbf{P}_{\mathrm{CO} 2} \\
\text { [bar] }\end{array}$} & \multirow{2}{*}{$\begin{array}{c}\mathrm{T} \\
{\left[{ }^{\circ} \mathrm{C}\right]}\end{array}$} & \multirow{2}{*}{$\begin{array}{c}t \\
{[\mathrm{~h}]}\end{array}$} & \multicolumn{5}{|c|}{$\begin{array}{c}\text { Si products / } \\
\text { Conversion [\%] }{ }^{[c]}\end{array}$} \\
\hline & & & & & & & a & b & c & d & $f+g$ \\
\hline 1 & $\begin{array}{l}\text { 1-Al } \\
(10)\end{array}$ & $\begin{array}{c}\mathrm{Et}_{3} \mathrm{Si}-\mathrm{H} \\
0\end{array}$ & $\mathrm{C}_{6} \mathrm{D}_{5} \mathrm{Br}$ & 1 & 80 & 48 & 0 & 0 & 0 & 0 & 0 \\
\hline 2 & $\begin{array}{c}\text { 1-Al } \\
(5.0) \\
\mathrm{B}\left(\mathrm{C}_{6} \mathrm{~F}_{5}\right)_{3} \\
(5.0)\end{array}$ & $\begin{array}{c}\mathrm{Et}_{3} \mathrm{Si}-\mathrm{H} \\
84\end{array}$ & $\mathrm{C}_{6} \mathrm{D}_{5} \mathrm{Br}$ & 1 & 80 & 5 & 0 & 4.7 & 0.2 & 74.3 & 2.7 \\
\hline 3 & $\begin{array}{c}\text { 1-Al } \\
(5.0) \\
\mathrm{B}\left(\mathrm{C}_{6} \mathrm{~F}_{5}\right)_{3} \\
(5.0)\end{array}$ & $\begin{array}{c}\mathrm{Et}_{3} \mathrm{Si}-\mathrm{H} \\
>98\end{array}$ & $\mathrm{C}_{6} \mathrm{D}_{5} \mathrm{Br}$ & 1 & 80 & 10 & 0 & 0 & 0.2 & 94.6 & 3.1 \\
\hline $4^{[\mathrm{d}]}$ & $\begin{array}{c}\text { 1-Al } \\
(5.0) \\
\mathrm{B}\left(\mathrm{C}_{6} \mathrm{~F}_{5}\right)_{3} \\
(5.0)\end{array}$ & $\begin{array}{c}\mathrm{Et}_{3} \mathrm{Si}-\mathrm{H} \\
>98\end{array}$ & $\mathrm{C}_{6} \mathrm{D}_{5} \mathrm{Br}$ & 6 & 80 & 5 & 0 & 28.6 & 0.3 & 53.7 & 7.2 \\
\hline 5 & $\begin{array}{c}\text { 1-Al } \\
(1.0) \\
\mathrm{B}\left(\mathrm{C}_{6} \mathrm{~F}_{5}\right)_{3} \\
(1.0) \\
\end{array}$ & $\begin{array}{c}\mathrm{Et}_{3} \mathrm{Si}-\mathrm{H} \\
54\end{array}$ & $\mathrm{C}_{6} \mathrm{D}_{5} \mathrm{Br}$ & 1 & 25 & 48 & 0 & 0.7 & 0.5 & 47.0 & 2.1 \\
\hline 6 & $\begin{array}{c}\text { 1-Al } \\
(1.0) \\
\mathrm{B}\left(\mathrm{C}_{6} \mathrm{~F}_{5}\right)_{3} \\
(1.0)\end{array}$ & $\begin{array}{c}\mathrm{Et}_{3} \mathrm{Si}-\mathrm{H} \\
27\end{array}$ & $\mathrm{C}_{6} \mathrm{D}_{6}$ & 1 & 25 & 48 & 0 & 2.6 & 0 & 17.4 & 2.4 \\
\hline
\end{tabular}




\begin{tabular}{|c|c|c|c|c|c|c|c|c|c|c|c|}
\hline $7^{[\mathrm{d}]}$ & $\begin{array}{c}\text { 1-Al } \\
(1.0) \\
\mathrm{B}\left(\mathrm{C}_{6} \mathrm{~F}_{5}\right)_{3} \\
(1.0) \\
\end{array}$ & $\begin{array}{c}\mathrm{Et}_{3} \mathrm{Si}-\mathrm{H} \\
>98\end{array}$ & $\mathrm{C}_{6} \mathrm{D}_{5} \mathrm{Br}$ & 6 & 25 & 30 & 0 & 1.2 & 0 & 91.6 & 2.9 \\
\hline $8^{[\mathrm{d}]}$ & $\begin{array}{c}\text { 1-Al } \\
(1.0) \\
\mathrm{B}\left(\mathrm{C}_{6} \mathrm{~F}_{5}\right)_{3} \\
(1.0)\end{array}$ & $\begin{array}{c}\mathrm{Et}_{3} \mathrm{Si}-\mathrm{H} \\
97\end{array}$ & $\mathrm{C}_{6} \mathrm{D}_{6}$ & 6 & 25 & 30 & 0 & 16.7 & 0.2 & 67.4 & 5.0 \\
\hline $9^{[\mathrm{d}]}$ & $\begin{array}{c}\text { 1-Al } \\
(1.0) \\
\mathrm{B}\left(\mathrm{C}_{6} \mathrm{~F}_{5}\right)_{3} \\
(1.0)\end{array}$ & $\begin{array}{c}\mathrm{Et}_{3} \mathrm{Si}-\mathrm{H} \\
>98\end{array}$ & $\mathrm{C}_{6} \mathrm{D}_{5} \mathrm{Br}$ & 6 & 80 & 5 & 0 & 40.1 & 1.9 & 46.0 & 4.8 \\
\hline $10^{[\mathrm{d}]}$ & $\begin{array}{c}\text { 1-Al } \\
(1.0) \\
\mathrm{B}\left(\mathrm{C}_{6} \mathrm{~F}_{5}\right)_{3} \\
(1.0)\end{array}$ & $\begin{array}{c}\mathrm{Et}_{3} \mathrm{Si}-\mathrm{H} \\
10\end{array}$ & $\mathrm{C}_{6} \mathrm{D}_{6}$ & 6 & 80 & 5 & 0 & 5.7 & 0 & 0.1 & 1.9 \\
\hline $11^{[\mathrm{d}]}$ & $\begin{array}{c}\text { 1-Al } \\
(1.0) \\
\mathrm{B}\left(\mathrm{C}_{6} \mathrm{~F}_{5}\right)_{3} \\
(1.0)\end{array}$ & $\begin{array}{c}\mathrm{Et}_{3} \mathrm{Si}-\mathrm{H} \\
>98\end{array}$ & $\mathrm{C}_{6} \mathrm{D}_{6}$ & 6 & 80 & 15 & 0 & 50.2 & 0.8 & 38.6 & 4.9 \\
\hline $12^{[\mathrm{d}]}$ & $\begin{array}{c}\text { 1-Al } \\
(0.5) \\
\mathrm{B}\left(\mathrm{C}_{6} \mathrm{~F}_{5}\right)_{3} \\
(1.5) \\
\end{array}$ & $\begin{array}{c}\mathrm{Et}_{3} \mathrm{Si}-\mathrm{H} \\
71\end{array}$ & $\mathrm{C}_{6} \mathrm{D}_{6}$ & 6 & 25 & 48 & 0 & 3.4 & 0 & 63.5 & 1.6 \\
\hline $13^{[\mathrm{d}]}$ & $\begin{array}{c}\text { 1-Al } \\
(1.5) \\
\mathrm{B}\left(\mathrm{C}_{6} \mathrm{~F}_{5}\right)_{3} \\
(0.5)\end{array}$ & $\begin{array}{c}\mathrm{Et}_{3} \mathrm{Si}-\mathrm{H} \\
96\end{array}$ & $\mathrm{C}_{6} \mathrm{D}_{6}$ & 6 & 25 & 48 & 0 & 30.0 & 0.7 & 53.9 & 4.5 \\
\hline
\end{tabular}




\begin{tabular}{|c|c|c|c|c|c|c|c|c|c|c|c|}
\hline $14^{[\mathrm{d}]}$ & $\begin{array}{c}\text { 1-Al } \\
(0.5) \\
\mathrm{B}\left(\mathrm{C}_{6} \mathrm{~F}_{5}\right)_{3} \\
(1.5)\end{array}$ & $\begin{array}{c}\mathrm{Et}_{3} \mathrm{Si}-\mathrm{H} \\
48\end{array}$ & $\mathrm{C}_{6} \mathrm{D}_{6}$ & 6 & 80 & 13 & 0 & 29.9 & 0.7 & 9.0 & 5.1 \\
\hline $15^{[\mathrm{d}]}$ & $\begin{array}{c}\text { 1-Al } \\
(1.5) \\
\mathrm{B}\left(\mathrm{C}_{6} \mathrm{~F}_{5}\right)_{3} \\
(0.5)\end{array}$ & $\begin{array}{c}\mathrm{Et}_{3} \mathrm{Si}-\mathrm{H} \\
90\end{array}$ & $\mathrm{C}_{6} \mathrm{D}_{6}$ & 6 & 80 & 13 & 0 & 60.1 & 0.8 & 11.6 & 8.7 \\
\hline 16 & $\begin{array}{c}\text { 1-Al } \\
(5.0) \\
\mathrm{Al}\left(\mathrm{C}_{6} \mathrm{~F}_{5}\right)_{3}(\text { toluene })_{0.5} \\
(5.0) \\
\end{array}$ & $\begin{array}{c}\mathrm{Et}_{3} \mathrm{Si}-\mathrm{H} \\
10\end{array}$ & $\mathrm{C}_{6} \mathrm{D}_{5} \mathrm{Br}$ & 1 & 80 & 5 & 1.6 & 0.5 & 1.1 & trace & 0 \\
\hline 17 & $\begin{array}{c}\text { 1-Al } \\
(5.0) \\
\mathrm{Al}\left(\mathrm{C}_{6} \mathrm{~F}_{5}\right)_{3}(\text { toluene })_{0.5} \\
(5.0) \\
\end{array}$ & $\begin{array}{c}\mathrm{Et}_{3} \mathrm{Si}-\mathrm{H} \\
15\end{array}$ & $\mathrm{C}_{6} \mathrm{D}_{5} \mathrm{Br}$ & 1 & 80 & 48 & 1.2 & 0.7 & 0 & trace & 0.5 \\
\hline $18^{[\mathrm{d}]}$ & $\begin{array}{c}\text { 1-Al } \\
(5.0) \\
\left.\mathrm{Al}\left(\mathrm{C}_{6} \mathrm{~F}_{5}\right)_{3} \text { (toluene }\right)_{0.5} \\
(5.0) \\
\end{array}$ & $\begin{array}{c}\mathrm{Et}_{3} \mathrm{Si}-\mathrm{H} \\
15\end{array}$ & $\mathrm{C}_{6} \mathrm{D}_{5} \mathrm{Br}$ & 6 & 80 & 5 & 1.4 & 0.4 & 2.8 & trace & 0 \\
\hline $19^{[\mathrm{d}]}$ & $\begin{array}{c}\text { 1-Al } \\
(5.0) \\
\mathrm{Al}\left(\mathrm{C}_{6} \mathrm{~F}_{5}\right)_{3}(\text { toluene })_{0.5} \\
(5.0)\end{array}$ & $\begin{array}{c}\mathrm{Et}_{3} \mathrm{Si}-\mathrm{H} \\
23\end{array}$ & $\mathrm{C}_{6} \mathrm{D}_{5} \mathrm{Br}$ & 6 & 80 & 48 & 0.9 & 0.8 & 1.0 & trace & 0 \\
\hline
\end{tabular}

${ }^{[a]}$ Reaction conditions, otherwise stated: solvent $(0.5 \mathrm{~mL}),[\mathrm{Si}-\mathrm{H}]_{0}=0.26 \mathrm{~mol} \cdot \mathrm{L}^{-1}$, [Cat $]_{0}=0.013 \mathrm{~mol} \cdot \mathrm{L}^{-1}, n\left(\mathrm{CO}_{2}\right)_{0}=1.3 \times 10^{-4}$ mol, internal standard (hexamethylbenzene) $=0.0616$ $\mathrm{mol} \cdot \mathrm{L}^{-1}$; ${ }^{[b]}$ Conversion of hydrosilane determined by the integration of the ${ }^{1} \mathrm{H}$ NMR resonances vs those of the standard, hexamethylbenzene; ${ }^{[c]}$ Yield of Si product determined by the integration of the corresponding ${ }^{1} \mathrm{H}$ NMR peak vs those of the standard, hexamethylbenzene. ${ }^{[\mathrm{d}]}$ solvent $(0.1 \mathrm{~mL}),[\mathrm{Si}-\mathrm{H}]_{0}=0.54 \mathrm{~mol} \cdot \mathrm{L}^{-1}$, $[\mathrm{Cat}]_{0}=0.054 \mathrm{~mol} \cdot \mathrm{L}^{-1}, n(\mathrm{CO})_{0}=$ $3.2 \times 10^{-4} \mathrm{~mol}$, internal standard (hexamethylbenzene) $=0.0615 \mathrm{~mol} \cdot \mathrm{L}^{-1}$. 
Table 5. Catalytic reduction of $\mathrm{CO}_{2}$ with different hydrosilanes in $\mathrm{C}_{6} \mathrm{D}_{6}$ at $80{ }^{\circ} \mathrm{C}$ and 6 bar in the presence of 1-Al/B(C $\left.\mathrm{C}_{6} \mathrm{~F}_{5}\right)_{3}\left([\mathrm{Al}]_{0}=[\mathrm{B}]_{0}=1 \mathrm{~mol} \%\right) .{ }^{[a]}$

\begin{tabular}{|c|c|c|c|c|c|c|c|}
\hline \multirow[t]{2}{*}{ Entry } & \multirow{2}{*}{$\begin{array}{c}\text { Silane / } \\
\text { Conversion } \\
{[\%]^{[b]}}\end{array}$} & \multirow{2}{*}{$\begin{array}{c}t \\
{[h]}\end{array}$} & \multicolumn{5}{|c|}{$\begin{array}{c}\text { Si products / } \\
\text { Conversion [\%] }{ }^{[\mathrm{cc}}\end{array}$} \\
\hline & & & a & b & c & d & $f+g$ \\
\hline 1 & $\begin{array}{c}\mathrm{Et}_{3} \mathrm{SiH} \\
10\end{array}$ & 5 & 0 & 5.7 & 0 & 0.1 & 1.9 \\
\hline 2 & $\begin{array}{c}\mathrm{PhMe}_{2} \mathrm{SiH} \\
75\end{array}$ & 5 & 0 & 0 & 0 & 56.1 & 5.8 \\
\hline 4 & $\begin{array}{c}\mathrm{PhSiH}_{3} \\
63\end{array}$ & 48 & 0 & 4.7 & 4.6 & 16.2 & 5.9 \\
\hline 3 & $\begin{array}{c}\mathrm{Ph}_{2} \mathrm{SiH}_{2} \\
35\end{array}$ & 48 & 0 & 31.1 & 0.8 & 0 & 1.1 \\
\hline 5 & $\begin{array}{c}\mathrm{Ph}_{3} \mathrm{SiH} \\
5\end{array}$ & 48 & 0 & 0 & 0 & 0 & 1.5 \\
\hline 6 & $\begin{array}{c}(\mathrm{EtO})_{3} \mathrm{SiH} \\
0\end{array}$ & 48 & 0 & 0 & 0 & 0 & 0 \\
\hline
\end{tabular}

${ }^{[a]}$ Reaction conditions, otherwise stated: solvent $(0.1 \mathrm{~mL}),[\mathrm{Si}-\mathrm{H}]_{0}=0.54 \mathrm{~mol} \cdot \mathrm{L}^{-1},[\mathrm{Cat}]_{0}=0.054 \mathrm{~mol} \cdot \mathrm{L}^{-1}, n\left(\mathrm{CO}_{2}\right)_{0}=3.2 \times 10^{-4}$ mol, internal standard (hexamethylbenzene) $=0.0615$ $\mathrm{mol} \cdot \mathrm{L}^{-1} ;{ }^{[b]}$ Conversion of hydrosilane determined by the integration of the ${ }^{1} \mathrm{H}$ NMR resonances vs those of the standard, hexamethylbenzene; ${ }^{[\mathrm{cc}}$ Yield of Si product determined by the integration of the corresponding ${ }^{1} \mathrm{H}$ NMR peak vs those of the standard, hexamethylbenzene. 
Within the series of combinations 1-M/B $\left(\mathrm{C}_{6} \mathrm{~F}_{5}\right)_{3}(\mathrm{M}=\mathrm{Ga}$, In and Zn; Table S3), the highest activity in hydrosilylation of $\mathrm{CO}_{2}$ with $\mathrm{Et}_{3} \mathrm{SiH}$ was observed with 1-In/ $\mathrm{B}\left(\mathrm{C}_{6} \mathrm{~F}_{5}\right)_{3}$. For instance, a result comparable with that of $\mathbf{1 - A l} / \mathrm{B}\left(\mathrm{C}_{6} \mathrm{~F}_{5}\right)_{3}$ (Table 4, entry 9) was obtained with $\mathbf{1}-\mathbf{I n} / \mathrm{B}\left(\mathrm{C}_{6} \mathrm{~F}_{5}\right)_{3}$ in $\mathrm{C}_{6} \mathrm{D}_{5} \mathrm{Br}$ at $80{ }^{\circ} \mathrm{C}$ and 6 bar $\mathrm{CO}_{2}$ pressure - a full conversion of hydrosilane within $13 \mathrm{~h}$ affording mostly $\mathbf{b}$ and $\mathbf{d}$ (Table S3, entry 8). In $\mathrm{C}_{6} \mathrm{D}_{6}$, the same reaction was less efficient resulting only in $43 \%$ of $\mathrm{Et}_{3} \mathrm{SiH}$ consumed (entry 9).

The binary 1-Zn/ $\mathrm{B}\left(\mathrm{C}_{6} \mathrm{~F}_{5}\right)_{3}$ system $\left([\mathrm{Zn}]_{0}=[\mathrm{B}]_{0}=5\right.$ mol\%) was also probed in $\mathrm{C}_{6} \mathrm{D}_{5} \mathrm{Br}$ at $80{ }^{\circ} \mathrm{C}$ and 1 or 6 bars of $\mathrm{CO}_{2}$ pressure (Table S3; entries 11-14). At higher pressure, 57\% conversion of hydrosilane was achieved within the first 5 hours (entry 13). However, the prolonged heating of the reaction resulted only in 67\% conversion at most after 2 days (entry 14), thus, suggesting deactivation of the catalytic system. Interestingly, among the three observed products of $\mathrm{CO}_{2}$ reduction (b-d), $\mathrm{Et}_{3} \mathrm{SiOCH}_{3}$ (c) was systematically the major one.

Kinetic and Mechanistic Studies of Hydrosilylation. Catalyst Transformation. A closer inspection of the NMR data obtained for the reaction mixtures through the series of catalytic tests (Tables 3-5 and S3) revealed a systematic presence of the two resonances in ca. 1:3 ratio in the aliphatic region of the corresponding ${ }^{1} \mathrm{H}$ NMR spectra: a broad ( $\delta 3.20$ or $\left.3.02 \mathrm{ppm}\right)$ and a sharp $(\delta$ 2.69 or $2.56 \mathrm{ppm}$ ) singlets in $\mathrm{C}_{6} \mathrm{D}_{5} \mathrm{Br}$ or $\mathrm{C}_{6} \mathrm{D}_{6}$, respectively. These two signals were assigned to the two compounds, $N$-silyl-anilines $\left(\mathrm{C}_{6} \mathrm{~F}_{5}\right) \mathrm{N}(H) \mathrm{SiEt}_{3}$ (f) and $\left(\mathrm{C}_{6} \mathrm{~F}_{5}\right) \mathrm{N}(\mathrm{Me}) \mathrm{SiEt}_{3}$ (g), in addition their nature was confirmed by GC-MS analysis (Figures S23 and S24, respectively).

To gain a better insight on a possible mechanism of hydrosilylation of $\mathrm{CO}_{2}$ with $\mathrm{Et}_{3} \mathrm{SiH}$ and to address the role of $\mathbf{f}$ and $\mathbf{g}$ in the catalytic process we carried out a series of kinetic studies with the most efficient tandem system 1-Al/ $B\left(\mathrm{C}_{6} \mathrm{~F}_{5}\right)_{3}$. Thus, the consumption of $\mathrm{Et}_{3} \mathrm{SiH}$, formation of 
products a-d and of compounds $\mathbf{f}$ and $\mathbf{g}$ were monitored by ${ }^{1} \mathrm{H}$ NMR spectroscopy under regular conditions (Figure 5) and also at different temperatures (Figures S25a-d). First, the reaction exhibited an induction period featuring a slow consumption of $\mathrm{Et}_{3} \mathrm{SiH}$, and a gradual formation of products b-d with a slow appearance of silyl-anilines $\mathbf{f}$ and $\mathbf{g}$. In previous studies on hydrosilylation of $\mathrm{CO}_{2},{ }^{15 a, 45}$ a similar induction period was observed and attributed ${ }^{15 a}$ to a slow process of the reorganization of the poorly active associated ion pair $\left[\mathrm{Cp}_{2}{ }_{2} \mathrm{Sc}\right]^{+}\left[\mathrm{HC}(\mathrm{O}) \mathrm{OB}\left(\mathrm{C}_{6} \mathrm{~F}_{5}\right)_{3}\right]^{-}$, derived from precatalyst $\left[\mathrm{Cp}_{2}{ }_{2} \mathrm{Sc}\right]^{+}\left[\mathrm{HB}\left(\mathrm{C}_{6} \mathrm{~F}_{5}\right)_{3}\right]^{-}$and $\mathrm{CO}_{2}$, into a significantly more catalytically efficient separated ion pair $\left[\mathrm{Cp}_{2}{ }_{2} \mathrm{Sc}(\mathbf{b})\right]^{+}\left[\mathrm{HC}(\mathrm{O}) \mathrm{OB}\left(\mathrm{C}_{6} \mathrm{~F}_{5}\right)_{3}\right]^{-}$, that is triggered in the presence of bis(silyl)acetal b. In our case, the existence of the induction period can also be related to a catalyst evolution process that is accompanied by elimination of silyl-anilines $\mathbf{f}$ and $\mathbf{g}$. In order to address this hypothesis, a kinetic experiment was performed using catalytic system $\mathbf{1 - A l} / \mathrm{B}_{(}\left(\mathrm{C}_{6} \mathrm{~F}_{5}\right)_{3}$ aged at room temperature in $\mathrm{C}_{6} \mathrm{D}_{6}$ for 12 hours in the presence of $\mathrm{Et}_{3} \mathrm{SiH}$. Prior to the addition of $\mathrm{CO}_{2}(6$ bars) the reaction featured ca. 19\% hydrosilane depletion and contained $4 \%$ of $\mathbf{f}$ and $\mathbf{g}$. The subsequent process proceeded at $80^{\circ} \mathrm{C}$ without induction period (Figure S27a) and, after 6 hours, resulted in formation of $\mathbf{b}$ with $70 \%$ yield and nearly complete conversion of hydrosilane.

After the initiation step, both the depletion of $\mathrm{Et}_{3} \mathrm{SiH}$ and the formation of $\mathbf{b}$ appeared to obey zero order kinetics (Figures S25b, c and d). This finding on the overall zero order in hydrosilane consumption parallels the behavior of $\left[\mathrm{Cp}_{2}^{*} \mathrm{Sc}\right]^{+}\left[\mathrm{HB}\left(\mathrm{C}_{6} \mathrm{~F}_{5}\right)_{3}\right]^{-}$precatalyst that was explained by the existence of a rate-determining step related to the formation of a key intermediate without an immediate participation of hydrosilane. ${ }^{15 a}$ The fact that in our case the rates of hydrosilane depletion and formation of $\mathbf{b}$ are not systematically equal and are both independent of the corresponding reactant/product concentrations may be diagnostic of a complicated multi-step mechanism operating with this catalytic system, where each reduction step can be catalyzed by a 
different species. Eyring analyses of the kinetic data (Figure S26) provided the corresponding free energy barrier value ( $\Delta G^{\neq} 298$ of $25(2)-27(2) \mathrm{kcal} \cdot \mathrm{mol}^{-1}$ ), which is comparable to those evaluated computationally for the prototype tandem $\mathrm{Al}\left(\mathrm{C}_{6} \mathrm{~F}_{5}\right)_{3} / \mathrm{B}\left(\mathrm{C}_{6} \mathrm{~F}_{5}\right)_{3}$ system $\left(\Delta G^{\neq}{ }_{298}\right.$ of $20-22$ $\left.\mathrm{kcal} \cdot \mathrm{mol}^{-1}\right){ }^{14}$

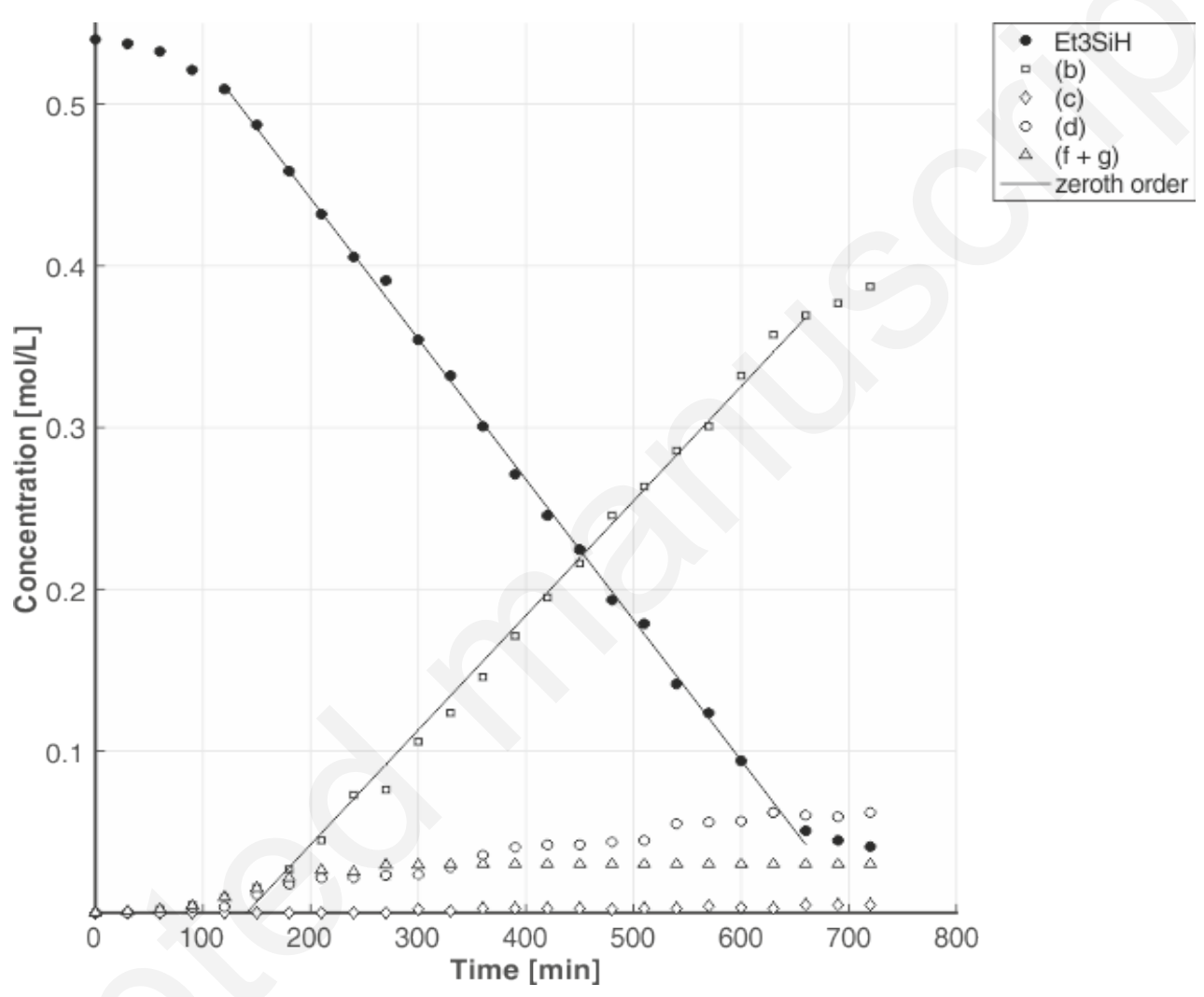

Figure 5. Kinetic plot of concentration $\left[\mathrm{mol} \cdot \mathrm{L}^{-1}\right.$ ] as a function of time [min] for reduction of $\mathrm{CO}_{2}$ with $\mathrm{Et}_{3} \mathrm{SiH}$ at $80{ }^{\circ} \mathrm{C}$ and 6 bar $\mathrm{CO}_{2}$ pressure: solvent $\mathrm{C}_{6} \mathrm{D}_{6}(0.1 \mathrm{~mL}),\left[\mathrm{Et}_{3} \mathrm{SiH}\right]_{0}=0.54 \mathrm{~mol} \cdot \mathrm{L}^{-1}$, $[\mathbf{1}-\mathrm{Al}]_{0}=0.054 \mathrm{~mol} \cdot \mathrm{L}^{-1},\left[\mathrm{~B}\left(\mathrm{C}_{6} \mathrm{~F}_{5}\right)_{3}\right]=0.054 \mathrm{~mol} \cdot \mathrm{L}^{-1}, n\left(\mathrm{CO}_{2}\right)=3.2 \times 10^{-4} \mathrm{~mol}$, internal standard (hexamethylbenzene) $=0.0615 \mathrm{~mol} \cdot \mathrm{L}^{-1} ; k_{\mathrm{app},[\mathrm{SiH}]}=1.45(7) \cdot 10^{-5} \mathrm{M} \cdot \mathrm{s}^{-1}, R^{2}=0.999, k_{\mathrm{app},[\mathbf{b}]}=$ $1.2(1) \cdot 10^{-5} \mathrm{M} \cdot \mathrm{s}^{-1}, R^{2}=0.994$.

The origin of silyl-anilines $\mathbf{f}$ and $\mathbf{g}$ was deduced from several independent stoichiometric experiments. First, addition of $\mathrm{B}\left(\mathrm{C}_{6} \mathrm{~F}_{5}\right)_{3}$ (1 equiv) to $\mathbf{1 - H}$ in $\mathrm{C}_{6} \mathrm{D}_{6}$ or in $\mathrm{C}_{6} \mathrm{D}_{5} \mathrm{Br}$ resulted in change of chemical shifts of the ${ }^{1} \mathrm{H}$ and ${ }^{19} \mathrm{~F}\left\{{ }^{1} \mathrm{H}\right\}$ NMR signals and their pattern for both reagents, which is 
in agreement with a possible formation of the $(\mathbf{1 - H}) \cdot B\left(\mathrm{C}_{6} \mathrm{~F}_{5}\right)_{3}$ adduct. ${ }^{46}$ Subsequent addition of excess $\mathrm{Et}_{3} \mathrm{SiH}$ after $20 \mathrm{~h}$ at $80{ }^{\circ} \mathrm{C}$ afforded a complete conversion of $\mathbf{1 - H}$ to an equimolar mixture of $\mathbf{f}$ and $\mathbf{g}$ (Figure S28). ${ }^{47}$ However, this pathway is unlikely the source of $\mathbf{f}$ and $\mathbf{g}$, provided the experiments were carried out under inert atmosphere and, thus, the preliminary hydrolysis of 1-Al can be excluded.

While no reaction was observed between 1-Al and $B\left(C_{6} F_{5}\right)_{3}$ in $C_{6} D_{5} B r$ or in $C_{6} D_{6}$ at $80{ }^{\circ} \mathrm{C}$, addition of $\mathrm{Et}_{3} \mathrm{SiH}$ (20 equiv) resulted in a complete consumption of $\mathrm{B}\left(\mathrm{C}_{6} \mathrm{~F}_{5}\right)_{3}$ and formation of an unidentified product (Figure S29). The same combination of $\mathbf{1 - A l}$ and $\mathrm{B}\left(\mathrm{C}_{6} \mathrm{~F}_{5}\right)_{3}$ and $\mathrm{Et}_{3} \mathrm{SiH}$ (20 equiv) in the presence of $\mathrm{CO}_{2}$ (20 equiv at 1 bar pressure) exhibited a complete consumption of hydrosilane after $20 \mathrm{~h}$ at $80{ }^{\circ} \mathrm{C}$ affording a complex mixture of unreacted 1-Al and $\mathrm{B}\left(\mathrm{C}_{6} \mathrm{~F}_{5}\right)$, silyl-anilines $\mathbf{f}$ and $\mathbf{g}, \mathrm{CH}_{4}$ (d) and some unidentified products (Figure S30). In an attempt to isolate intermediates of this reduction reaction the corresponding reaction mixture was subjected to crystallization in toluene. Gratifyingly, a new compound - the $\mathrm{B}\left(\mathrm{C}_{6} \mathrm{~F}_{5}\right)_{3}$-adduct of $N$-silyl-formamidine $\mathbf{1}-\mathrm{SiEt}_{3}\left(\left\{\mathrm{~B}\left(\mathrm{C}_{6} \mathrm{~F}_{5}\right)_{3}\right\} \cdot \mathbf{1}-\mathrm{SiEt}_{3}\right)^{48}$ was isolated, whose identity was established by X-ray diffraction analysis (Figure 6). On the other hand, all attempts to obtain exploitable NMR data for the crystals of this compound failed due to its remarkable instability in solution. ${ }^{49}$ The formation of $\left\{\mathrm{B}\left(\mathrm{C}_{6} \mathrm{~F}_{5}\right)_{3}\right\} \cdot \mathbf{1}-\mathbf{S i E t}_{3}$ is likely resulted from the putative process of a $\mathrm{B}\left(\mathrm{C}_{6} \mathrm{~F}_{5}\right)_{3}$-assisted reduction of 1-Al with $\mathrm{Et}_{3} \mathrm{SiH}$ leading to 1-SiEt $\mathbf{t}_{3}$ and an unidentified aluminum hydrido species, followed by coordination of the former with $\mathrm{B}\left(\mathrm{C}_{6} \mathrm{~F}_{5}\right)_{3}{ }^{22,46,50}$ All attempts to isolate and authenticate aluminum-containing intermediates and products of this reaction were unsuccessful so far.

It should be mentioned that in the above reactions the formation of such typical products of

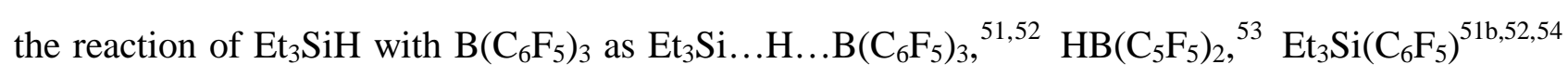


and $\left\{\mathrm{B}\left(\mathrm{C}_{6} \mathrm{~F}_{5}\right)_{3}\right\} \cdot \mathrm{Et}_{3} \mathrm{SiOC}(\mathrm{O}) \mathrm{H}^{14}$ either was not observed or could not be unequivocally corroborated.

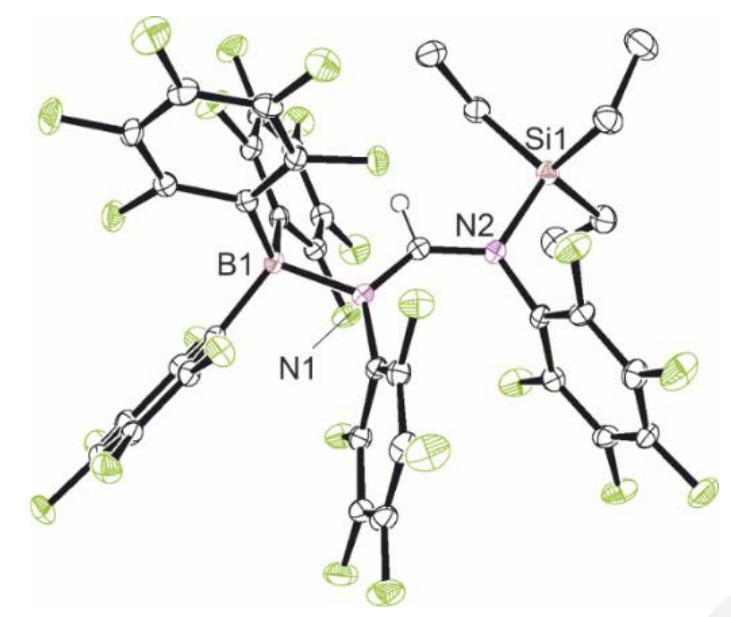

Figure 6. Molecular structure of $\left\{\mathrm{B}\left(\mathrm{C}_{6} \mathrm{~F}_{5}\right)_{3}\right\} \cdot \mathbf{1}-\mathrm{SiEt}_{3}$.

A series of $\mathrm{CO}_{2}$ hydrosilylation experiments was attempted using proligand $\mathbf{1 - H}$ in combination with $\mathrm{B}\left(\mathrm{C}_{6} \mathrm{~F}_{5}\right)_{3}$ or $\mathrm{Al}\left(\mathrm{C}_{6} \mathrm{~F}_{5}\right)_{3}$ (toluene $)_{0.5}$ (Table 6). The hydrosilylation reaction was not operational in the presence of single $\mathbf{1 - H}$ (entry 1), whereas upon addition of $B\left(C_{6} F_{5}\right)_{3}$ as cocatalyst, a nearly one-half conversion of hydrosilane was achieved over $24 \mathrm{~h}$ at $80{ }^{\circ} \mathrm{C}$ and 6 bar $\mathrm{CO}_{2}$ pressure both in $\mathrm{C}_{6} \mathrm{D}_{5} \mathrm{Br}$ and in $\mathrm{C}_{6} \mathrm{D}_{6}$ (entries 3 and 4, respectively). However, the metal-free system 1-H/B $\left(\mathrm{C}_{6} \mathrm{~F}_{5}\right)_{3}$ appeared to be substantially less efficient than 1-Al/B $\left(\mathrm{C}_{6} \mathrm{~F}_{5}\right)_{3}$ (compare with entries 9 and 11 in Table 4, respectively) also providing larger amounts of methane (d) in these experiments. In all experiments, formation of significant amounts of anilines $\mathbf{f}$ and $\mathbf{g}$ was detected, apparently derived from the reduction processes. Under the same experimental conditions, 1-H/Al $\left(\mathrm{C}_{6} \mathrm{~F}_{5}\right)_{3}$ (toluene $)_{0.5}$ congener was found completely inactive (entry 5). Also, the in situ combination of $\mathbf{1 - S i E t} \mathbf{t}_{3} / \mathrm{B}\left(\mathrm{C}_{6} \mathrm{~F}_{5}\right)_{3}$, probed under the same conditions $\left(\mathrm{C}_{6} \mathrm{D}_{6}, 80{ }^{\circ} \mathrm{C}, 6\right.$ bar $)$ as those enabling the higher performances with $\mathbf{1 - A l} / \mathrm{B}\left(\mathrm{C}_{6} \mathrm{~F}_{5}\right)_{3}$ (Table 4 , entry 11), resulted only in $23 \%$ conversion of hydrosilane after 48 hours (entry 6) giving methane as the principal product (17\%). 
The above observations suggest that the binary catalytic systems obtained upon combining 1-M $(\mathrm{M}=\mathrm{B}, \mathrm{Al}, \mathrm{Ga}, \mathrm{In}, \mathrm{Zn})$ with $\mathrm{E}\left(\mathrm{C}_{6} \mathrm{~F}_{5}\right)_{3}$ are susceptible of reacting with $\mathrm{Et}_{3} \mathrm{SiH}$, thus, releasing 1-SiEt $_{3}$ which then follows a prompt reduction affording products $\mathbf{f}$ and $\mathbf{g}$. These two $\mathrm{N}$-silyl-anilines, as well as $\mathbf{1 - S i E t _ { 3 }}$ itself, in combination with $\mathrm{B}\left(\mathrm{C}_{6} \mathrm{~F}_{5}\right)_{3}$ can also constitute catalytically active systems capable of $\mathrm{CO}_{2}$ hydrosilylation reaction as tandem FLP-like $\mathrm{R}_{3} \mathrm{~N} / \mathrm{B}\left(\mathrm{C}_{6} \mathrm{~F}_{5}\right)_{3}$ analogues. $^{7}$ On the other hand, the nature of other Al-containing products, derived from 1-Al, that are apparently responsible for the predominant formation of $\mathbf{b}$, could not be established so far. 
Table 6. Catalytic reduction of $\mathrm{CO}_{2}$ with $\mathrm{Et}_{3} \mathrm{SiH}$ in the presence of $\mathbf{1 - H}$ and $\mathbf{1}-\mathbf{S i E t}_{3}{ }^{\text {[a] }}$

\begin{tabular}{|c|c|c|c|c|c|c|c|c|c|c|c|}
\hline \multirow[t]{2}{*}{ Entry } & \multirow{2}{*}{$\begin{array}{c}\text { Precat } \\
\text { [mol\%] }\end{array}$} & \multirow{2}{*}{$\begin{array}{c}\text { Silane / } \\
\text { Conversion } \\
{[\%]^{[b]}}\end{array}$} & \multirow[t]{2}{*}{ Solvent } & \multirow{2}{*}{$\begin{array}{c}\mathbf{P}_{\mathrm{CO} 2} \\
\text { [bar] }\end{array}$} & \multirow{2}{*}{$\begin{array}{c}\mathbf{T} \\
{\left[{ }^{\circ} \mathbf{C}\right]}\end{array}$} & \multirow{2}{*}{$\begin{array}{c}t \\
{[h]}\end{array}$} & \multicolumn{5}{|c|}{$\begin{array}{c}\text { Si products / } \\
\text { Conversion [\%] }{ }^{[c]}\end{array}$} \\
\hline & & & & & & & $\mathbf{a}$ & b & c & d & $\mathbf{f}+\mathbf{g}$ \\
\hline $1^{[\mathrm{d}]}$ & $\begin{array}{c}\mathbf{1 - H} \\
(10.0)\end{array}$ & $\begin{array}{c}\mathrm{Et}_{3} \mathrm{Si}-\mathrm{H} \\
0\end{array}$ & $\mathrm{C}_{6} \mathrm{D}_{5} \mathrm{Br}$ & 6 & 80 & 48 & 0 & 0 & 0 & 0 & 0 \\
\hline 2 & $\begin{array}{c}\mathbf{1 - H} \\
(6.0) \\
\mathrm{B}\left(\mathrm{C}_{6} \mathrm{~F}_{5}\right)_{3} \\
(1.0)\end{array}$ & $\begin{array}{c}\mathrm{Et}_{3} \mathrm{Si}-\mathrm{H} \\
21\end{array}$ & $\mathrm{C}_{6} \mathrm{D}_{5} \mathrm{Br}$ & 1 & 80 & 24 & 0 & 0.6 & 0 & 0 & 10.0 \\
\hline $3^{[\mathrm{d}]}$ & $\begin{array}{c}\mathbf{1 - H} \\
(6.0) \\
\mathrm{B}\left(\mathrm{C}_{6} \mathrm{~F}_{5}\right)_{3} \\
(1.0)\end{array}$ & $\begin{array}{c}\mathrm{Et}_{3} \mathrm{Si}-\mathrm{H} \\
44\end{array}$ & $\mathrm{C}_{6} \mathrm{D}_{5} \mathrm{Br}$ & 6 & 80 & 24 & 0 & 1.6 & 0 & 20.1 & 7.4 \\
\hline $4^{[\mathrm{d}]}$ & $\begin{array}{c}\mathbf{1 - H} \\
(6.0) \\
\mathrm{B}\left(\mathrm{C}_{6} \mathrm{~F}_{5}\right)_{3} \\
(1.0)\end{array}$ & $\begin{array}{c}\mathrm{Et}_{3} \mathrm{Si}-\mathrm{H} \\
49\end{array}$ & $\mathrm{C}_{6} \mathrm{D}_{6}$ & 6 & 80 & 24 & 0 & 3.8 & 0 & 26.4 & 6.4 \\
\hline $5^{[\mathrm{d}]}$ & $\begin{array}{c}\text { 1-H } \\
(6.0) \\
\operatorname{Al}\left(\mathrm{C}_{6} \mathrm{~F}_{5}\right)_{3}(\mathrm{tol})_{0.5} \\
(1.0)\end{array}$ & $\begin{array}{c}\mathrm{Et}_{3} \mathrm{Si}-\mathrm{H} \\
0\end{array}$ & $\mathrm{C}_{6} \mathrm{D}_{5} \mathrm{Br}$ & 6 & 80 & 24 & 0 & 0 & 0 & 0 & 0 \\
\hline $6^{[\mathrm{d}]}$ & $\begin{array}{c}\mathbf{1 - S i E t} t_{3} \\
(1.0) \\
\mathrm{B}\left(\mathrm{C}_{6} \mathrm{~F}_{5}\right)_{3} \\
(1.0)\end{array}$ & $\begin{array}{c}\mathrm{Et}_{3} \mathrm{Si}-\mathrm{H} \\
23\end{array}$ & $\mathrm{C}_{6} \mathrm{D}_{6}$ & 6 & 80 & 48 & 0 & 3.9 & 0 & 17.3 & 0.3 \\
\hline
\end{tabular}

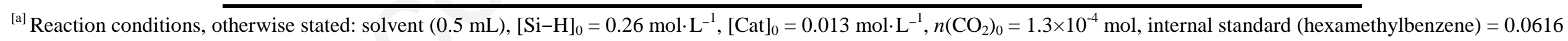

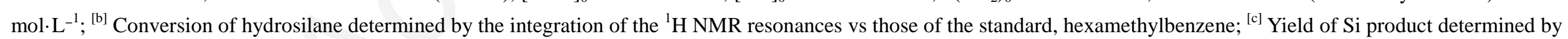


the integration of the corresponding ${ }^{1} \mathrm{H} \mathrm{NMR}$ peak vs those of the standard, hexamethylbenzene; ${ }^{[\mathrm{d}]}$ solvent $(0.1 \mathrm{~mL}),[\mathrm{Si}-\mathrm{H}]_{0}=0.54 \mathrm{~mol} \cdot \mathrm{L}^{-1}$, $[\mathrm{Cat}]_{0}=0.054 \mathrm{~mol} \cdot \mathrm{L}^{-1}, n\left(\mathrm{CO}{ }_{2}\right)=$ $3.2 \times 10^{-4} \mathrm{~mol}$, internal standard (hexamethylbenzene) $=0.0615 \mathrm{~mol} \cdot \mathrm{L}^{-1}$. 


\section{CONCLUSIONS}

In this study, a series of homoleptic group 13 (1-B, 1-Al, 1-Ga, 1-In) and group 12 (1-Zn) complexes incorporating electron-withdrawing formamidinate ligands was synthesized. Their solution and solid-state structures were established using NMR techniques and XRD analysis, respectively. The higher Lewis acidic character of $\mathbf{1 - B}$ as compared to that of $\mathrm{B}\left(\mathrm{C}_{6} \mathrm{~F}_{5}\right)_{3}$ was established using both experimental and computational techniques.

The obtained complexes were studied as precursors for $\mathrm{CO}_{2}$ reduction using hydrosilanes as reducing agents. While single-component 1-M exhibited no or very poor activity in hydrosilylation, the two-component systems derived from 1-M and $\mathrm{E}\left(\mathrm{C}_{6} \mathrm{~F}_{5}\right)_{3}(\mathrm{E}=\mathrm{B}, \mathrm{Al})$ were found significantly more active. Among them, a binary combination $\mathbf{1 - A l} / \mathrm{B}\left(\mathrm{C}_{6} \mathrm{~F}_{5}\right)_{3}$ showed the best performance within the whole series, thus providing high hydrosilane $\left(\mathrm{Et}_{3} \mathrm{SiH}\right)$ conversions under a range of conditions ( $\mathrm{P}_{\mathrm{CO} 2}$, temperature, benzene or bromobenzene solvents). The main products of these reactions are $\mathrm{CH}_{2}\left(\mathrm{OSiEt}_{3}\right)_{2}$ (b) and $\mathrm{CH}_{4}(\mathbf{d})$, while the product ratio was found to be dependent on the reaction conditions. Other hydrosilanes $\left(\mathrm{PhSiMe}_{2} \mathrm{H}, \mathrm{PhSiH}_{3}\right.$ and $\left.\mathrm{Ph}_{2} \mathrm{SiH}_{2}\right)$ were also successfully employed for $\mathrm{CO}_{2}$ hydrosilylation with 1-Al/ $\mathrm{B}\left(\mathrm{C}_{6} \mathrm{~F}_{5}\right)_{3}$ under the optimized condition.

Transformation of the initial catalytic precursors affording multiple species was evidenced by kinetic and mechanistic studies. In particular, the hydrosilane/B( $\left(\mathrm{C}_{6} \mathrm{~F}_{5}\right)_{3}$-assisted cleavage of the ligand-metal bond in the formamidinate precursor 1-M $(\mathrm{M}=\mathrm{Al})$ affording $\mathrm{N}$-silyl-formamidine 1-SiEt $t_{3}$ is surmised to be one of the key steps towards the formation of unidentified so far aluminum-based species featuring a better catalytic performance in hydrosilylation of $\mathrm{CO}_{2}$. At the same time, different $\mathrm{N}$-bases, namely $\left(\mathrm{C}_{6} \mathrm{~F}_{5}\right) \mathrm{N}(\mathrm{H}) \mathrm{SiEt}_{3}$ and $\left(\mathrm{C}_{6} \mathrm{~F}_{5}\right) \mathrm{N}(\mathrm{Me}) \mathrm{SiEt}_{3}$, concomitantly generated from the precursor on the initiation step can also participate as the FLP partners to 
$\mathrm{B}\left(\mathrm{C}_{6} \mathrm{~F}_{5}\right)_{3}$ and, thus, interfere as cocatalysts in several different reduction steps of hydrosilylation. Understanding the behavior of a catalytic system and factors (steric and electronic) responsible for its stability and performance will allow rational engineering of new more efficient and selective molecular catalysts for chemical transformations of $\mathrm{CO}_{2}$. These investigations are underway in our labs.

\section{EXPERIMENTAL SECTION}

General Considerations. All manipulations were performed under a purified argon atmosphere using standard Schlenk techniques or in a glovebox. Solvents were distilled from $\mathrm{Na} /$ benzophenone (THF, $\mathrm{Et}_{2} \mathrm{O}$ ) and $\mathrm{Na} / \mathrm{K}$ alloy (toluene, pentane) under argon, degassed thoroughly and stored under argon prior to use. Deuterated solvents $\left(\mathrm{C}_{6} \mathrm{D}_{6}, \mathrm{C}_{7} \mathrm{D}_{8}\right.$, THF- $d_{8} ;>99.5 \% \mathrm{D}$, Deutero $\mathrm{GmbH}$ and Euroisotop) were vacuum-transferred from $\mathrm{Na} / \mathrm{K}$ alloy into storage tubes. $\mathrm{CDCl}_{3}$, $\mathrm{CD}_{2} \mathrm{Cl}_{2}$ and $\mathrm{C}_{6} \mathrm{D}_{5} \mathrm{Br}$ were kept over $\mathrm{CaH}_{2}$ and vacuum-transferred before use. $\mathrm{CO}_{2}$ (Air Liquide, 99.99\%) was additionally dried over molecular sieves (Sertronic $500 \mathrm{~T} \mathrm{DB}$ ) prior to utilization. Base-free $\mathrm{B}\left(\mathrm{C}_{6} \mathrm{~F}_{5}\right)_{3}$ was obtained by sublimation of the adduct $\left(\mathrm{Et}_{2} \mathrm{O}\right) \cdot \mathrm{B}\left(\mathrm{C}_{6} \mathrm{~F}_{5}\right)_{3}{ }^{55}$ under high dynamic vacuum. $\mathrm{Al}\left(\mathrm{C}_{6} \mathrm{~F}_{5}\right)_{3}$ (toluene $)_{0.5},{ }^{56} \mathbf{1 - H},{ }^{23} \mathbf{a},{ }^{14} \mathbf{b}^{57}$ and $\mathbf{c}^{14}$ were prepared according to literature protocols. Other starting materials were purchased from Alfa, Strem, Acros or Aldrich, and used as received.

Instruments and Measurements. NMR spectra of complexes were recorded on Bruker AM-400 and AM-500 spectrometers in Teflon-valved NMR tubes at $25^{\circ} \mathrm{C}$, unless otherwise indicated. ${ }^{1} \mathrm{H}$ NMR chemical shifts are reported in ppm vs $\mathrm{SiMe}_{4}$ using the residual solvent resonances. ${ }^{19} \mathrm{~F}$ NMR chemical shifts were determined by external reference to an aqueous solution of $\mathrm{NaBF}_{4} \cdot{ }^{11} \mathrm{~B} \mathrm{NMR}$ spectra were referenced to external $\mathrm{BF}_{3} \cdot \mathrm{OEt}_{2}$. Assignment of resonances was 
made from $2 \mathrm{D}{ }^{1} \mathrm{H}-{ }^{1} \mathrm{H}$ COSY, ${ }^{1} \mathrm{H}-{ }^{13} \mathrm{C}$ HSQC and HMBC NMR experiments. Coupling constants are given in Hertz. Elemental analyses (C, H, N) were performed using a Flash EA1112 CHNS Thermo Electron apparatus and are the average of two independent determinations.

Synthesis of $\mathbf{B}(\mathbf{N C N})_{3}$. In the glovebox, 1-H (5.02 g, $\left.13.3 \mathrm{mmol}\right)$ and potassium hydride (0.80 g, 19.9 mmol) were placed into a Schlenk tube equipped with a magnetic stirrer. Dry THF (30 mL) was added under argon and the reaction mixture was stirred at room temperature. After $12 \mathrm{~h}$, the reaction mixture was filtered and all the volatiles were removed under vacuum. The crude potassium salt of the ligand was dried under vacuum for $4 \mathrm{~h}$, and dry $\mathrm{Et}_{2} \mathrm{O}$ (30 mL) was added followed by addition of $\mathrm{BCl}_{3}\left(4.45 \mathrm{~mL}, 13.3 \mathrm{mmol}\right.$, a $1 \mathrm{M}$ solution in $\left.\mathrm{CH}_{2} \mathrm{Cl}_{2}\right)$. The mixture was stirred at room temperature overnight and then filtered and concentrated to a half of the initial volume. The solid was crystallized at room temperature by addition to this solution of dry heptane (4 mL). Off-white crystals were separated and dried under vacuum to afford pinkish powder of 1-B (2.36 g, $2.1 \mathrm{mmol}, 80 \%) .{ }^{1} \mathrm{H}$ NMR $\left(\mathrm{C}_{6} \mathrm{D}_{6}, 400 \mathrm{MHz}, 25{ }^{\circ} \mathrm{C}\right): \delta 8.27$ (s, 3H, CH). ${ }^{19} \mathrm{~F}\left\{{ }^{1} \mathrm{H}\right\}$ NMR $\left(\mathrm{C}_{6} \mathrm{D}_{6}, 376.5 \mathrm{MHz}, 25^{\circ} \mathrm{C}\right): \delta-148.55$ (br m, 12F, $\mathrm{C}_{6} F_{5}$ ), -155.75 (br m, 6F, $\mathrm{C}_{6} F_{5}$ ), -162.18 (br m, 12F, $\left.\mathrm{C}_{6} \mathrm{~F}_{5}\right) .{ }^{13} \mathrm{C}\left\{{ }^{1} \mathrm{H}\right\} \mathrm{NMR}\left(\mathrm{CD}_{2} \mathrm{Cl}_{2}, 400 \mathrm{MHz}, 25{ }^{\circ} \mathrm{C}\right.$ ) (some signals from quaternary carbons were not identified): $\delta 153.4$ (s, CH), 142.3 (br d, ${ }^{1} J=250, o-C_{6} \mathrm{~F}_{5}$ ), 140.5 (br d, ${ }^{1} \mathrm{~J}=255, p-C_{6} \mathrm{~F}_{5}$ ), 139.1 (br d, ${ }^{1} J=250, m-C_{6} \mathrm{~F}_{5}$ ). Anal.Calcd for $\mathrm{C}_{39} \mathrm{H}_{3} \mathrm{BF}_{30} \mathrm{~N}_{6}$ : C, 41.23; H, 0.27; N, 7.40. Found: C, 41.16; H, 0.26; N, 7.33.

Synthesis of $\mathbf{A l}(\mathbf{N C N})_{3}$ (1-Al). To a solution of $\mathbf{1 - H}(1.0 \mathrm{~g} 2.6 \mathrm{mmol})$ in toluene $(5 \mathrm{~mL})$ was added trimethylaluminium (1 M solution in heptane, $0.89 \mathrm{~mL}, 0.89 \mathrm{mmol}$ ). The reaction mixture was stirred at room temperature overnight. All volatiles were evaporated in vacuo, and the crude product was recrystallized from toluene to give white crystals of 1-Al (0.89 g, 0.77 mmol, 87\%). ${ }^{1} \mathrm{H}$ $\operatorname{NMR}\left(\mathrm{C}_{6} \mathrm{D}_{6}, 400 \mathrm{MHz}, 25^{\circ} \mathrm{C}\right): \delta 7.75$ (s, 3H, CH). ${ }^{19} \mathrm{~F}\left\{{ }^{1} \mathrm{H}\right\}$ NMR $\left(\mathrm{C}_{6} \mathrm{D}_{6}, 376.5 \mathrm{MHz}, 25^{\circ} \mathrm{C}\right): \delta-$ 
$153.45\left(\mathrm{~d}, J=20.7,12 \mathrm{~F}, o-\mathrm{C}_{6} F_{5}\right),-159.85\left(\mathrm{t}, J=22.6,6 \mathrm{~F}, p-\mathrm{C}_{6} F_{5}\right),-162.88(\mathrm{t}, J=42.1,12 \mathrm{~F}$, $\left.m-\mathrm{C}_{6} F_{5}\right) .{ }^{13} \mathrm{C}\left\{{ }^{1} \mathrm{H}\right\}$ NMR $\left(\mathrm{CD}_{2} \mathrm{Cl}_{2}, 400 \mathrm{MHz}, 25^{\circ} \mathrm{C}\right.$ ) (some signals from quaternary carbons were not identified): $\delta 153.1$ (s, CH), 141.4 (br d, ${ }^{1} J=247, o-C_{6} \mathrm{~F}_{5}$ ), 138.6 (br d, ${ }^{1} J=251, m-C_{6} \mathrm{~F}_{5}$ ). Anal.Calcd for $\mathrm{C}_{39} \mathrm{H}_{3} \mathrm{AlF}_{30} \mathrm{~N}_{6}$ : C, 40.65; H, 0.26; N, 7.29. Found: C, 40.62; H, 0.25; N, 7.23.

Synthesis of $\mathbf{G a}(\mathbf{N C N})_{3}$ (1-Ga). Using a protocol similar to that described for 1-B, compound 1-Ga was obtained from 1-H (3.0 g $7.8 \mathrm{mmol}), \mathrm{KH}(0.50 \mathrm{~g} 13 \mathrm{mmol})$ and $\mathrm{GaCl}_{3}(0.47 \mathrm{~g} 2.6 \mathrm{mmol})$. 1-Ga was isolated as a slightly pink solid $(0.37 \mathrm{~g}, 0.31 \mathrm{mmol}, 12 \%) .{ }^{1} \mathrm{H}$ NMR $\left(\mathrm{C}_{6} \mathrm{D}_{6}, 400 \mathrm{MHz}\right.$, $\left.25^{\circ} \mathrm{C}\right): \delta 7.66(\mathrm{~s}, 3 \mathrm{H}, \mathrm{CH}) .{ }^{19} \mathrm{~F}\left\{{ }^{1} \mathrm{H}\right\} \operatorname{NMR}\left(\mathrm{C}_{6} \mathrm{D}_{6}, 376.5 \mathrm{MHz}, 25^{\circ} \mathrm{C}\right): \delta-153.28(\mathrm{~d}, J=22.0,12 \mathrm{~F}$, $\left.o-C_{6} F_{5}\right),-159.86\left(\mathrm{t}, J=22.0,6 \mathrm{~F}, p-\mathrm{C}_{6} F_{5}\right),-162.88\left(\mathrm{t}, J=22.0,12 \mathrm{~F}, m-\mathrm{C}_{6} F_{5}\right)$. Numerous attempts to obtain the ${ }^{13} \mathrm{C}$ NMR data for this compound failed; only signals from solvent were observed in the corresponding NMR spectra. Anal.Calcd for $\mathrm{C}_{39} \mathrm{H}_{3} \mathrm{GaF}_{30} \mathrm{~N}_{6}$ : C, 39.19; $\mathrm{H}, 0.25 ; \mathrm{N}, 7.03$. Found: C, 39.22; H, 0.27; N, 7.14.

Synthesis of $\operatorname{In}(\mathbf{N C N})_{3}$ (1-In). Using a protocol similar to that described for 1-B, compound 1-In was obtained from 1-H (3.0 g $7.8 \mathrm{mmol}), \mathrm{KH}(0.50 \mathrm{~g} 13 \mathrm{mmol})$ and $\mathrm{InBr}_{3}(0.92 \mathrm{~g} 2.6 \mathrm{mmol})$. 1-In was isolated as a pink powder (0.36g, $0.29 \mathrm{mmol}, 11 \%) .{ }^{1} \mathrm{H} \mathrm{NMR}\left(\mathrm{C}_{6} \mathrm{D}_{6}, 400 \mathrm{MHz}, 25{ }^{\circ} \mathrm{C}\right): \delta$ 8.20 (s, 3H, CH). ${ }^{19} \mathrm{~F}\left\{{ }^{1} \mathrm{H}\right\}$ NMR $\left(\mathrm{C}_{6} \mathrm{D}_{6}, 376.5 \mathrm{MHz}, 25{ }^{\circ} \mathrm{C}\right): \delta-154.23$ (d, $\left.J=21.4,12 \mathrm{~F}, \mathrm{C}_{6} F_{5}\right),-$ $160.69\left(\mathrm{t}, J=21.4,6 \mathrm{~F}, \mathrm{C}_{6} F_{5}\right),-162.77\left(\mathrm{t}, J=21.4,12 \mathrm{~F}, \mathrm{C}_{6} F_{5}\right) .{ }^{13} \mathrm{C}\left\{{ }^{1} \mathrm{H}\right\} \mathrm{NMR}\left(\mathrm{CD}_{2} \mathrm{Cl}_{2}, 400 \mathrm{MHz}\right.$, $25^{\circ} \mathrm{C}$ ): $\delta 164.6(\mathrm{~s}, \mathrm{CH}), 142.0\left(\mathrm{br} \mathrm{d},{ }^{1} J=250, o-C_{6} \mathrm{~F}_{5}\right.$ ), 140.5 (br d, ${ }^{1} J=255, m-C_{6} \mathrm{~F}_{5}$ ), 137.0 (br d, ${ }^{1} J=250, p-C_{6} \mathrm{~F}_{5}$ ), 119.9 (br s, ipso- $C_{6} \mathrm{~F}_{5}$ ). Anal.Calcd for $\mathrm{C}_{39} \mathrm{H}_{3} \mathrm{InF}_{30} \mathrm{~N}_{6}:$ C, 37.77; H, 0.24; N, 6.78. Found: C, 38.01; H, 0.29; N, 6.98.

Synthesis of $\mathbf{Z n}(\mathbf{N C N})_{2}(\mathbf{1 - Z n})$. Using a protocol similar to that described for 1-Al, compound 1-Zn was obtained from 1-H (3.0 g $7.8 \mathrm{mmol}_{\text {), }} \mathrm{Et}_{2} \mathrm{Zn}(1 \mathrm{M}$ in heptane, $2.67 \mathrm{~mL}, 2.67 \mathrm{mmol})$ and isolated as transparent crystalline solid (1.8 g, $2.1 \mathrm{mmol}, 83 \%) .{ }^{1} \mathrm{H}$ NMR $\left(\mathrm{C}_{6} \mathrm{D}_{6}, 400 \mathrm{MHz}, 25{ }^{\circ} \mathrm{C}\right)$ : 
$\delta 7.93$ (s, 2H, CH) ppm. ${ }^{19} \mathrm{~F}\left\{{ }^{1} \mathrm{H}\right\}$ NMR $\left(\mathrm{C}_{6} \mathrm{D}_{6}, 376.5 \mathrm{MHz}, 25{ }^{\circ} \mathrm{C}\right): \delta-152.97$ (br m, 8F, $\left.\mathrm{C}_{6} F_{5}\right),-$ 160.04 (br m, 4F, $\mathrm{C}_{6} F_{5}$ ), -162.41 (t, $\left.J=20.8,8 \mathrm{~F}, \mathrm{C}_{6} F_{5}\right) .{ }^{13} \mathrm{C}\left\{{ }^{1} \mathrm{H}\right\}$ NMR $\left(\mathrm{C}_{6} \mathrm{D}_{6}, 400 \mathrm{MHz}, 25{ }^{\circ} \mathrm{C}\right.$ ) (some signals from quaternary carbons were not identified): $\delta 169.03$ (s, CH), 141.8 (br d, ${ }^{1} \mathrm{~J}=246$, $o-C_{6} \mathrm{~F}_{5}$ ), 137.9 (br d, ${ }^{1} J=248, m-C_{6} \mathrm{~F}_{5}$ ), 120.6 (br s, ipso- $C_{6} \mathrm{~F}_{5}$ ). Anal.Calcd for $\mathrm{C}_{26} \mathrm{H}_{2} \mathrm{~F}_{20} \mathrm{~N}_{4} \mathrm{Zn}$ : C, 38.28; H, 0.25; N, 6.87. Found: C, 38.34; H, 0.30; N, 7.01.

Synthesis of $\mathbf{E t}_{3} \mathbf{S i}(\mathbf{N C N})$ (1-SiEt $\left.\mathbf{t}_{3}\right)$. In the glovebox, 1-H (0.50 g, $\left.1.3 \mathrm{mmol}\right)$ and KH (0.09 g, $2.0 \mathrm{mmol}$ ) were placed into a Schlenk tube equipped with a magnetic stirrer. Dry THF (15 mL) was added under argon and the reaction mixture was stirred at room temperature. After $12 \mathrm{~h}$, the reaction mixture was filtered and all the volatiles were removed under vacuum. The crude potassium salt was dried under vacuum for $4 \mathrm{~h}$, and dry toluene $(15 \mathrm{~mL})$ was added followed by addition of $\mathrm{Et}_{3} \mathrm{SiCl}(2.25 \mathrm{~mL}, 1.3 \mathrm{mmol})$ at $-78{ }^{\circ} \mathrm{C}$. The mixture was stirred overnight at room temperature and then filtered. All volatiles were removed and a brownish oily product was obtained, and then dried under vacuum overnight to give 1-SiEt $\mathbf{3}_{\mathbf{3}}(0.03 \mathrm{~g}, 0.55 \mathrm{mmol}, 42 \%)$. The compound apparently exists as a ca. 1:0.9 mixture of two atropoisomers arising from a congested rotation around the C-N single bond (Figure S19), ${ }^{58}$ which slowly interconvert at room temperature. ${ }^{1} \mathrm{H}$ NMR $\left(\mathrm{C}_{7} \mathrm{D}_{8}, 400\right.$ $\mathrm{MHz}, 25^{\circ} \mathrm{C}$ ): $\delta 7.66$ (br s, $1 \mathrm{H}, \mathrm{CH}$ ), 0.86 (br m, 9H, $\mathrm{CH}_{3} \mathrm{CH}_{2}$ ), 0.59 (br m, $6 \mathrm{H}, \mathrm{CH}_{3} \mathrm{CH}_{2}$ ). ${ }^{19} \mathrm{~F}\left\{{ }^{1} \mathrm{H}\right\}$ NMR ( ${ }_{7} \mathrm{D}_{8}, 376.5 \mathrm{MHz}, 25^{\circ} \mathrm{C}$ ): $\delta-145.9$ (br m $\mathrm{C}_{6} F_{5}$ ), -155.2 (br m, $\mathrm{C}_{6} F_{5}$ ), -155.5 (t, $J=18.8$, $\mathrm{C}_{6} F_{5}$ ), -162.8 (br m, $\mathrm{C}_{6} F_{5}$ ), -164.6 (m, $\left.\mathrm{C}_{6} F_{5}\right) .{ }^{13} \mathrm{C}\left\{{ }^{1} \mathrm{H}\right\}$ NMR $\left(\mathrm{C}_{7} \mathrm{D}_{8}, 400 \mathrm{MHz}, 25{ }^{\circ} \mathrm{C}\right.$ ) (some signals from quaternary carbons were not identified): $\delta 156.6$ (br s, $1 \mathrm{C}, \mathrm{CH}$ ), 144.9 (br d, ${ }^{1} \mathrm{~J}=246$, $o-C_{6} \mathrm{~F}_{5}$ ), 141.1(br d, $\left.{ }^{1} J=251, o-C_{6} \mathrm{~F}_{5}\right), 140.8$ (br d, ${ }^{1} J=251, p-C_{6} \mathrm{~F}_{5}$ ), $138.3\left(\mathrm{br} \mathrm{d},{ }^{1} J=251, m-C_{6} \mathrm{~F}_{5}\right.$ ), 137.3 (br d, $\left.{ }^{1} J=251, p-C_{6} \mathrm{~F}_{5}\right), 6.4\left(\mathrm{~s}, \mathrm{CH}_{3} \mathrm{CH}_{2}\right), 4.0\left(\mathrm{~s}, \mathrm{CH}_{3} \mathrm{CH}_{2}\right.$ ). Despite repeated attempts, reproducible and satisfactory elemental analysis for $\mathbf{1 - S i E t _ { 3 }}\left(\mathrm{C}_{19} \mathrm{H}_{16} \mathrm{~F}_{10} \mathrm{~N}_{2} \mathrm{Si}\right)$ could not be obtained, apparently due to the extreme air- and moisture-sensitivity of the compound, and possible formation 
during pyrolysys of non- pyrolisable silicon carbide.

General procedure for $\mathrm{CO}_{2}$ hydrosilylation - NMR-scale reactions. (A) Reaction at 1 bar $\mathrm{CO}_{2}$ pressure. In the glovebox, a Teflon-valved NMR tube was charged with the internal standard hexamethylbenzene (5.0 mg, $0.0308 \mathrm{mmol}), \mathrm{HSiEt}_{3}(21 \mu \mathrm{L}, 0.13 \mathrm{mmol})$, pre-catalyst $\left(6.15 \times 10^{-3}\right.$ mmol; $5.0 \mathrm{~mol} \%$ vs hydrosilane) and, when relevant, a solution of $\mathrm{E}\left(\mathrm{C}_{6} \mathrm{~F}_{5}\right)_{3}(5.0 \mathrm{~mol} \%$ vs hydrosilane). Then, dry degassed $\mathrm{C}_{6} \mathrm{D}_{5} \mathrm{Br}$ or $\mathrm{C}_{6} \mathrm{D}_{6}(0.5 \mathrm{~mL})$ was added, and the NMR tube was sealed. The tube was degassed by two freeze-pump-thaw cycles and charged with pure $\mathrm{CO}_{2}$ under 1 bar pressure $(0.13 \mathrm{mmol})$. Afterwards, it was introduced in the NMR spectrometer preset at the desired temperature. The progress of the reaction was monitored by NMR spectroscopy.

(B) Reaction at 6 bar $\mathrm{CO}_{2}$ pressure. In the glovebox, a Wilmad® high-pressure Teflon-valved NMR tube was charged with the internal standard hexamethylbenzene (1.0 mg, $0.00616 \mathrm{mmol}$ ), $\mathrm{HSiEt}_{3}(8.6 \mu \mathrm{L}, 0.054 \mathrm{mmol})$, a diluted solution of pre-catalyst $\left(5.4 \times 10^{-4} \mathrm{mmol} ; 1.0 \mathrm{~mol} \%\right.$ vs hydrosilane), relevant $\mathrm{E}\left(\mathrm{C}_{6} \mathrm{~F}_{5}\right)_{3}\left(1.0\right.$ mol\% vs hydrosilane), and dry degassed $\mathrm{C}_{6} \mathrm{D}_{5} \mathrm{Br}$ or $\mathrm{C}_{6} \mathrm{D}_{6}(0.1$ $\mathrm{mL}$ ). Then the NMR tube was sealed. The tube was degassed by two freeze-pump-thaw cycles and charged with pure $\mathrm{CO}_{2}$ under 6 bar pressure $(0.32 \mathrm{mmol})$. Afterwards, it was introduced in the NMR spectrometer preset at the desired temperature. The progress of the reaction was monitored by NMR spectroscopy.

Computational Details. The calculations were performed using the Gaussian $09^{59}$ program employing B3PW91 ${ }^{60}$ functional, and using a standard double- $\xi$ polarized basis set, namely the 6-31(d,p) set. The solvent effects, in our case for toluene, were taken into account during all the calculations by means of the SMD model. ${ }^{61}$ All stationary points were fully characterized via analytical frequency calculations as either true minima (all positive eigenvalues) or transition states (one imaginary eigenvalue). The IRC procedure was used to confirm the nature of each transition 
state connecting two minima. ${ }^{62}$ Zero-point vibrational energy corrections (ZPVE) were estimated by a frequency calculation at the same level of theory, to be considered for the calculation of the total energy values. The electronic charges (at the DFT level) were computed using the natural population analysis (NPA). ${ }^{63}$

Crystal Structure Determination of 1-H, 1-B, 1-Al, 1-Ga, 1-In, 1-Zn and $\left\{\mathbf{B}\left(\mathbf{C}_{\mathbf{6}} \mathbf{F}_{5}\right)_{3}\right\}\left(\mathbf{1}-\mathbf{S i E t}_{3}\right)$. Diffraction data were collected at $100 \mathrm{~K}$ using a Bruker APEX CCD diffractometer with graphite-monochromatized MoK $\alpha$ radiation $(\lambda=0.71073 \AA)$. A combination of $\omega$ and $\theta$ scans was carried out to obtain a unique data set. The crystal structures were solved by direct methods, remaining atoms were located from difference Fourier synthesis followed by full-matrix least-squares refinement based on F2 (programs SIR97 and SHELXL-97). ${ }^{64}$ Many hydrogen atoms could be located from the Fourier difference analysis. Other hydrogen atoms were placed at calculated positions and forced to ride on the attached atom. The hydrogen atom positions were calculated but not refined. All non-hydrogen atoms were refined with anisotropic displacement parameters. Crystal data and details of data collection and structure refinement for the different compounds are given in Table S1. Crystal data, details of data collection and structure refinement for all compounds (CCDC 1950208-1950214, respectively) can be obtained from the Cambridge Crystallographic Data Centre via www.ccdc.cam.ac.uk/data_request/cif.

\section{ASSOCIATED CONTENT}

Supporting information is available free of charge on the ACS Publications website at DOI: Selected catalytic results, NMR data, kinetic plots (PDF). 


\section{AUTHOR INFORMATION}

\section{Corresponding Authors}

*E-mail for E.K.: evgueni.kirillov@univ-rennes1.fr

\section{ORCID}

Evgueni Kirillov: 0000-0002-5067-480X

Notes

The authors declare no competing financial interest.

\section{ACKNOWLEDGEMENTS}

The authors of this work thank ANR-17-CE06-0006-01 “CO22CHEM” for financial support. W.H. is grateful to the International Master of Molecular Catalysis and Green Chemistry program of the University of Rennes 1.

\section{REFERENCES}

1 For recent reviews see: (a) Martin, R.; Tortajada, A.; Juliá-Hernández, F.; Borjesson, M.; Moragas, T. Transition-Metal-Catalyzed Carboxylation Reactions with Carbon Dioxide. 
Angew. Chem., Int. Ed. 2018, 57, 15948-15982; (b) Pramudita, R. A.; Motokura, K. Transformative reduction of carbon dioxide through organocatalysis with silanes. Green Chem. 2018, 20, 4834-4843; (c) Tappe, N. A.; Reich, R. M.; D'Elia, V.; Kühn, F. E. Current advances in the catalytic conversion of carbon dioxide by molecular catalysts: an update. Dalton Trans. 2018, 47, 13281-13313; (d) Fernandez-Alvarez, F. J.; Oro, L. A. Homogeneous Catalytic Reduction of $\mathrm{CO}_{2}$ with Silicon - Hydrides, State of the Art. ChemCatChem 2018, 10, 4783-4796.

Olah, G. A.; Beyond oil and gas: the methanol economy. Angew. Chem., Int. Ed. 2005, 44, 2636-2639.

For some representative articles see: (a) Jansen, A.; Görls, H.; Pitter, S. Trans-[ $\left.\mathrm{Ru}^{\mathrm{II}} \mathrm{Cl}(\mathrm{MeCN})_{5}\right]\left[\mathrm{Ru}^{\mathrm{III}} \mathrm{Cl}_{4}(\mathrm{MeCN})_{2}\right]$ : A reactive intermediate in the homogeneous catalyzed hydrosilylation of carbon dioxide. Organometallics 2000, 19, 135-138. (b) Jansen, A.; Stephan, P. Homogeneously catalyzed reduction of carbon dioxide with silanes: a study on solvent and ligand effects and catalyst recycling. J. Mol. Catal. A: Chem. 2004, 217, 41-45. (c) Park, S.; Bezier, D.; Brookhart, M. An Efficient Catalyst for Reduction of Carbon Dioxide to Methane with Trialkylsilanes. J. Am. Chem. Soc. 2012, 134, 11404-11407. (d) Zhang, L.; Cheng, J.; Hou, Z. Highly efficient catalytic hydrosilylation of carbon dioxide by an N-heterocyclic carbene copper catalyst. Chem. Commun. 2013, 49, 4782-4784. (e) Itagaki, S.; Yamaguchi, K.; Mizuno, N. Catalytic synthesis of silyl formates with 1 atm of $\mathrm{CO}_{2}$ and their utilization for synthesis of formyl compounds and formic acid. J. Mol. Catal. A: Chem. 2013, 366, 347-352. (f) Metsänen, T. T.; Oestreich, M.; Temperature-dependent chemoselective hydrosilylation of carbon dioxide to formaldehyde or methanol oxidation state. 
Organometallics 2014, 34, 543-546. (g) Jurado-Vázquez, T.; Ortiz-Cervantes, C.; García, J. J.

Catalytic reduction of $\mathrm{CO}_{2}$ with organo-silanes using $\left[\mathrm{Ru}_{3}(\mathrm{CO})_{12}\right]$. J. Organomet. Chem. 2016, 823, 8-13. (h) Takaya, J.; Iwasawa, N. Synthesis, structure, and catalysis of palladium complexes bearing a group 13 metalloligand: remarkable effect of an aluminum-metalloligand in hydrosilylation of $\mathrm{CO}_{2}$. J. Am. Chem. Soc. 2017, 139, 6074-6077. (i) Nakamae, K.; Tanaka, M.; Kure, B.; Nakajima, T.; Ura, Y.; Tanase, T. A fluxional $\mathrm{Cu}_{8} \mathrm{H}_{6}$ cluster supported by bis(diphenylphosphino)methane and its facile reaction with $\mathrm{CO}_{2}$. Chem. Eur. J. 2017, 23, 9457-9461. (k) Jurado-Vázquez, T.; García, J. J. Iron Catalyzed $\mathrm{CO}_{2}$ Activation with Organosilanes. Catal. Lett. 2018, 148, 1162-1168. (l) Bertini, F.; Glatz, M.; Stoger, B.; Peruzzini, M.; Veiros, L. F.; Kirchner, K.; Gonsalvi, L. Carbon Dioxide Reduction to Methanol Catalyzed by Mn(I) PNP Pincer Complexes under Mild Reaction Conditions. ACS Catal. 2019, 9, 632-639. (m) Guzmán, J.; García-Orduña, P.; Polo, V.; Lahoz, F. J.; Oro, L. A.; Fernández-Alvarez, F. J. Ir-catalyzed selective reduction of $\mathrm{CO}_{2}$ to the methoxy or formate level with $\mathrm{HSiMe}\left(\mathrm{OSiMe}_{3}\right)_{2}$. Catal. Sci. Technol. 2019, 9, 2858-2867.

(a) Sattler, W.; Parkin, G. Zinc Catalysts for On-Demand Hydrogen Generation and Carbon Dioxide Functionalization. J. Am. Chem. Soc. 2012, 134, 17462-17465. (b) Rit, A.; Zanardi, A.; Spaniol, T. P.; Maron,L.; Okuda, J. A Cationic Zinc Hydride Cluster Stabilized by an N-Heterocyclic Carbene: Synthesis, Reactivity, and Hydrosilylation Catalysis. Angew. Chem. Int. Ed. 2014, 53, 13273-13277. (c) Tüchler, M.; Gärtner, L.; Fischer, S.; Boese, A. D.; Belaj, F.; Mösch-Zanetti, N. C. Efficient $\mathrm{CO}_{2}$ Insertion and Reduction Catalyzed by a Terminal Zinc Hydride Complex. Angew. Chem. Int. Ed. 2018, 57, 6906-6909. (d) Feng, G.; Du, C.; Xiang, L.; Li, Rosal, I. del G.; Leng, X.; Chen, Y. Side arm twist on Zn-catalyzed 
hydrosilylative reduction of $\mathrm{CO}_{2}$ to formate and methanol equivalents with high selectivity and activity. ACS Catal. 2018, 8, 4710-4718. (e) Bruyere, J.-C.; Specklin, D.; Gourlaouen, C.; Lapenta, R.; Veiros, L. F.; Grassi, A.; Milione, A.; Ruhlman, L.; Boudon, C.; Dagorne, S. Chem. Eur. J. 2019, 25, 8061-8069.

(a) Khandelwal, M.; Wehmschulte, R. J. Deoxygenative reduction of carbon dioxide to methane, toluene, and diphenylmethane with $\left[\mathrm{Et}_{2} \mathrm{Al}\right]^{+}$as catalyst. Angew. Chem. Int. Ed. 2012, 51, 7323-7326. (b) Saleh, M.; Powell, D. R.; Wehmschulte, R. J. Catalytic Reduction of Carbon Dioxide Using Cationic Organoaluminum and-Gallium Compounds. Organometallics, 2017, 36, 4810-4815. (c) Dagorne, S.; Wehmschulte, R. Recent Developments on the Use of Group 13 Metal Complexes in Catalysis. ChemCatChem 2018, 10, 2509-2520, $\mathrm{CO}_{2}$ Catalyzed by Triphenylborane in Highly Polar, Aprotic Solvents. Chem. Eur. J. 2016, 22, 7730-7733.

(a) Stephan, D. W.; Erker, G. Frustrated Lewis pair chemistry of carbon, nitrogen and sulfur oxides. Chem. Sci. 2014, 5, 2625-2641. (b) Fontaine, F.-G.; Courtemanche, M.-A.; Legare, M.-A.; Rochette, E. Design principles in frustrated Lewis pair catalysis for the functionalization of carbon dioxide and heterocycles. Coord. Chem. Rev. 2017, 334, 124-135.

Berkefeld, A.; Piers, W. E.; Parvez, M. Tandem Frustrated Lewis Pair/Tris(pentafluorophenyl)borane-Catalyzed Deoxygenative Hydrosilation of Carbon Dioxide. J. Am. Chem. Soc. 2010, 132, 10660-10661.

9 (a) Courtemanche, M.-A.; Legare, M.-A.; Rochette, E.; Fontaine, F.-G. Phosphazenes: efficient organocatalysts for the catalytic hydrosilylation of carbon dioxide. Chem. Commun. 2015, 51, 
6858-6861. (b) Chong, C. C.; Kinjo, R. Hydrophosphination of $\mathrm{CO}_{2}$ and Subsequent Formate

Transfer in the 1,3,2 - Diazaphospholene - Catalyzed N-Formylation of Amines. Angew. Chem. Int. Ed. 2015, 54, 12116-12120.

Motokura, K.; Nakagawa, C.; Pramudita, R. A.; Manaka, Y. Formate-Catalyzed Selective Reduction of Carbon Dioxide to Formate Products using Hydrosilanes. ACS Sustain. Chem. Eng. 2019, 7, 11056-11061

Pramudita, R. A.; Motokura, K. Transformative reduction of carbon dioxide through organocatalysis with silanes. Green Chem. 2018, 20, 4834-4843.

Rauch, M.; Strater, Z.; Parkin, G. Selective Conversion of Carbon Dioxide to Formaldehyde via a Bis(silyl)acetal: Incorporation of Isotopically Labeled $\mathrm{C}_{1}$ Moieties Derived from Carbon Dioxide into Organic Molecules. J. Am. Chem. Soc. 2019, 141, 17754-17762.

(a) Specklin, D.; Hild, F.; Fliedel, C.; Gourlaouen, C.; Veiros, L. F.; Dagorne, S. Accessing Two-Coordinate $\mathrm{Zn}{ }^{\mathrm{II}}$ Organocations by NHC Coordination: Synthesis, Structure, and Use as $\pi$ Lewis Acids in Alkene, Alkyne, and $\mathrm{CO}_{2}$ Hydrosilylation. Chem. Eur. J. 2017, 23, 15908-15912; (b) Rauch, M.; Parkin, G. Zinc and magnesium catalysts for the hydrosilylation of carbon dioxide. J. Am. Chem. Soc. 2017, 139, 18162-18165; (c) Zhang, Q.; Fukaya, N.; Fujitani, T.; Choi, J. C. Carbon Dioxide Hydrosilylation to Methane Catalyzed by Zinc and Other First-Row Transition Metal Salts. Bull. Chem. Soc. Jpn. 2019, 92, 1945-1949.

Chen, J.; Falivene, L.; Caporaso, L.; Cavallo, L.; Chen, E. Y. X. Selective reduction of $\mathrm{CO}_{2}$ to $\mathrm{CH}_{4}$ by tandem hydrosilylation with mixed Al/B catalysts. J. Am. Chem. Soc. 2016, 138, $5321-5333$.

(a) Berkefeld, A.; Piers, W. E.; Parvez, M. ; Castro, L. ; Maron, L. ; Eisenstein, O. 
Decamethylscandocinium-hydrido-(perfluorophenyl)-borate: fixation and tandem tris(perfluorophenyl)-borane catalyzed Deoxygenative hydrosilation of carbon dioxide. Chem. Sci. 2013, 4, 2152-2162; (b) LeBlanc, F. A.; Piers, W. E.; Parvez, M. Selective Hydrosilation of $\mathrm{CO}_{2}$ to a Bis(silylacetal) Using an Amido Bipyridyl-Ligated Organoscandium Catalyst. Angew. Chem. Int. Ed. 2014, 53, 789-792. (c) Beh, D. W.; Piers, W. E.; Gelfand, B. S.; Lin, J.-B. Tandem Deoxygenative hydrosilation of carbon dioxide with a cationic scandium hydridoborate and $\mathrm{B}\left(\mathrm{C}_{6} \mathrm{~F}_{5}\right)_{3}$. Chem. Eur. J. 2020, 49, 95-101.

(a) Matsuo, T.; Kawaguchi, H. From Carbon Dioxide to Methane: Homogeneous Reduction of Carbon Dioxide with Hydrosilanes Catalyzed by Zirconium-Borane Complexes. J. Am. Chem. Soc. 2006, 128, 12362-12363; (b) Luconi, L.; Rossin, A.; Tuci, G.; Gafurov, Z.; Lyubov, D. M.; Trifonov, A. A.; Cicchi, S.; Ba, H.; Pham-Huu, C.; Yakhvarov, D.; Giambastiani, G. Benzoimidazole-Pyridylamido Zirconium and Hafnium Alkyl Complexes as Homogeneous Catalysts for Tandem Carbon Dioxide Hydrosilylation to Methane. ChemCatChem, 2019, 11, 495-510.

Jiang, Y.; Blacque, O.; Fox, T.; Berke, H. Catalytic $\mathrm{CO}_{2}$ Activation Assisted by Rhenium Hydride/B $\left(\mathrm{C}_{6} \mathrm{~F}_{5}\right) 3$ Frustrated Lewis Pairs-Metal Hydrides Functioning as FLP Bases. J. Am. Chem. Soc. 2013, 135, 7751-7760.

(a) Mitton, S. J.; Turculet, L. Mild Reduction of Carbon Dioxide to Methane with Tertiary Silanes Catalyzed by Platinum and Palladium Silyl Pincer Complexes. Chem. Eur. J. 2012, 18, 15258-15262; (b) González-Sebastián, L.; Flores-Alamo, M.; García, J. J. Nickel-catalyzed hydrosilylation of $\mathrm{CO}_{2}$ in the presence of $\mathrm{Et}_{3} \mathrm{~B}$ for the synthesis of formic acid and related formates. Organometallics 2013, 32, 7186-7194; (c) Huang, X.; Zhang, K.; Shao, Y.; Li, Y., 
Gu, F.; Qu, L. B.; Ke, Z. Mechanism of Si-H Bond Activation for Lewis Acid PBP-Ni-Catalyzed Hydrosilylation of $\mathrm{CO}_{2}$ : The Role of the Linear SN2 Type Cooperation. ACS Catal. 2019, 9, 5279-5289.

Edelmann, F.T. Recent progress in the Chemistry of Metal Amidinates and Guanidinates: Synthesis, Catalysis and Materials in Advances in Organometallic Chemistry. (Eds.: Hill, A.F.; Fink, M.J.), Elsevier, 2013, pp 55-374.

(a) Dureen, M. A.; Stephan, D. W. Reactions of Boron Amidinates with $\mathrm{CO}_{2}$ and $\mathrm{CO}$ and Other Small Molecules. J. Am. Chem. Soc. 2010, 132, 13559-13568. (b) Cabrera, A. R.; Rojas, R. S.; Valderrama, M.; Pluss, P.; Berke, H.; Daniliuc, C. G.; Kehr, G.; Erker, G. Synthesis of new asymmetric substituted boron amidines-reactions with CO and transfer hydrogenations of phenylacetylene. Dalton Trans. 2015, 44, 19606-19614. (c) Yang, L.; Ren, X.; Wang, H.; Zhang, N.; Hong, S. The reaction mechanisms of boron amidinate and small molecules: a density function theory study. Res. Chem. Intermed. 2012, 38, 113-133.

(a) Moreno, S.; Ramos, A.; Carrillo-Hermosilla, F.; Rodríguez-Diéguez, A.; García-Vivó, D.; Fernández-Galán, R.; Antiñolo, A. Selective Three-Component Coupling for $\mathrm{CO}_{2}$ Chemical Fixation to Boron Guanidinato Compounds. Inorg. Chem. 2018, 57, 8404-8413. (b) Aders, N.; Keweloh, L.; Pleschka, D.; Hepp, A.; Layh, M.; Rogel, F.; Uhl, W. P-H Functionalized Al/P-Based Frustrated Lewis Pairs in Dipolar Activation and Hydrophosphination: Reactions with $\mathrm{CO}_{2}$ and $\mathrm{SO}_{2}$. Organometallics 2019, 38, 2839-2852. (c) Szynkiewicz, N.; Ordyszewska, A.; Chojnacki, J.; Grubba, R. Diaminophosphinoboranes: effective reagents for phosphinoboration of $\mathrm{CO}_{2}$. RSC Adv. 2019, 9, 27749-27753. 
Perfluoroarylboranes. Inorg. Chem. 2011, 50, 12252-12262.

Abdou, H. E.; Mohamed, A.A.; López-de-Luzuriaga, J. M.; Fackler, J. P. Tetranuclear Gold(I)

Clusters with Nitrogen Donor Ligands: Luminescence and X-Ray Structure of Gold(I) Naphthyl Amidinate Complex. J. Clust. Sci. 2004, 15, 397-411.

The molecular structure of $\mathbf{1 - H} \cdot($ toluene) was reported in: Krackl, S.; Inoue, S.; Driess, M.;

Enthaler, S. Intermolecular Hydrogen-Fluorine Interaction in Dimolybdenum Triply Bonded Complexes Modified by Fluorinated Formamidine Ligands for the Construction of 2D- and 3D-Networks. Eur. J. Inorg. Chem. 2011, 2103-2111.

Birkmann, B.; Voss, T.; Geier, S. J.; Ullrich, M.; Kehr, G.; Erker, G.; Stephan, D. W. Frustrated Lewis pairs and ring-opening of THF, dioxane, and thioxane. Organometallics 2010, 29, $5310-5319$.

(a) Weetman, C.; Hill, M. S.; Mahon, M. F. Magnesium Catalysis for the Hydroboration of Carbodiimides. Chem. Eur. J. 2016, 22, 7158-7162. (b) Liu, H.; Kulbitski, K.; Tamm, M.; Eisen, M. S. Organoactinide-Catalyzed Monohydroboration of Carbodiimines. Chem. Eur. J. 2018, 24, 5738-5742.

Protchenko, A. V.; Urbano, J.; Abdalla, J. A. B. ; Campos, J. ; Vidovic, D. ; Schwarz, A. D. ; Blake, M. P. ; Mountford, P. ; Jones, C.; Aldridge, S. Electronic Delocalization in Two and Three Dimensions: Differential Aggregation in Indium "Metalloid” Clusters. Angew. Chem.Int. Ed. 2017, 56, 15098-15102.

Braun, U.; Habereder, T.; Noth, H.; Piotrowski, H.; Warchhold, M. Triaminoboranes and Their Metallation to N - Lithiotriaminoboranes. Eur. J. Inorg. Chem. 2002, 1132-1145. 
centers: $\mathrm{Al}^{3+}, 0.535 \AA \mathrm{Ga}^{3+}, 0.620 \AA \AA \mathrm{In}^{3+}, 0.800$. Å. Shannon, R. D. Revised effective ionic radii and systematic studies of interatomic distances in halides and chalcogenides. Acta Crystallogr., Sect. A. 1976, A32, 751-767.

Bayram, M.; Blaser, D.; Wolper, C.; Schulz, S. Syntheses and Structures of Bis-AmidinateAlane Complexes. Organometallics 2014, 33, 2080-2087.

(a) Dagorne, S.; Guzei, I. A.; Coles, M. P.; Jordan, R. F. Synthesis and Structures of Cationic Aluminum and Gallium Amidinate Complexes. J. Am. Chem. Soc. 2000, 122, 274-289. (b) Jones, C.; Junk, P. C.; Kloth, M.; Proctor, K. M.; Stasch, A. Bulky amidinato complexes and amidine adducts of Al, Ga and In halides. Polyhedron 2006, 25, 1592-1600.

(a) Tsai, Y.-J.; Lo, W.; Zhao, Q. Ligand control in nuclearity of Zn complexes supported by formamidinates. Polyhedron 2015, 97, 39-46. (b) Schmidt, S.; Schulz, S.; Blaser, D.; Boese, R.; Bolte, M. Synthesis and Structural Characterization of New Zinc Amidinate Complexes. Organometallics 2010, 29, 6097-6103.

Due to poor solubility of this compound at low temperature no comprehensive NMR data could be collected.

Laszlo, P.; Teston, M. Determination of the acidity of Lewis acids. J. Am. Chem. Soc. 1990, 112, 8750-8754.

Beckett, M. A.; Strickland, G. C.; Holland, J. R.; Varma, S. A convenient NMR method for the measurement of Lewis acidity at boron centres: correlation of reaction rates of Lewis acid initiated epoxide polymerizations with Lewis acidity. Polymer 1996, 37, 4629- 4631.

Heiden, Z. M.; Thompson, B. L., Influence of Lewis Acid Strength on Molecule Activation. In The Essential Guide to Lewis Acids, (Ed.: Sandes, O. M.), Nova Science Publishers, Inc.: 
Hauppauge, New York, 2019; pp 1-87.

Britovsek, G. J. P.; Ugolotti, J.; White, A. J. P. From $B\left(\mathrm{C}_{6} \mathrm{~F}_{5}\right)_{3}$ to $\mathrm{B}\left(\mathrm{OC}_{6} \mathrm{~F}_{5}\right)_{3}$ : Synthesis of $\left(\mathrm{C}_{6} \mathrm{~F}_{5}\right)_{2} \mathrm{BOC}_{6} \mathrm{~F}_{5}$ and $\mathrm{C}_{6} \mathrm{~F}_{5} \mathrm{~B}\left(\mathrm{OC}_{6} \mathrm{~F}_{5}\right)_{2}$ and their relative Lewis acidity. Organometallics, 2005, 24, 1685-1691.

Sivaev, I. B.; Bregadze, V. I. Lewis acidity of boron compounds. Coord. Chem. Rev. 2014, 270, 75-88.

The amounts of $\mathrm{CO}_{2}\left(n\left(\mathrm{CO}_{2}\right)_{0}\right)$, used for the reactions in sealed NMR tubes, were estimated from the ideal gas law equation.

The following signals in the corresponding ${ }^{1} \mathrm{H}$ NMR spectra $\left(25{ }^{\circ} \mathrm{C}, \mathrm{C}_{6} \mathrm{D}_{6}\right.$ or $\mathrm{C}_{6} \mathrm{D}_{5} \mathrm{Br}$, respectively) were used as references: $\mathrm{HC}(\mathrm{O}) \mathrm{OSiEt}_{3}(\delta 7.76$ and $7.95 \mathrm{ppm}), \mathrm{H}_{2} \mathrm{C}(\mathrm{O}) \mathrm{OSiEt}_{3}(\delta$ 5.06 and $5.10 \mathrm{ppm}), H_{3} \mathrm{COSiEt}_{3}(\delta 3.32$ and $3.34 \mathrm{ppm})$ and $\mathrm{CH}_{4}(\delta 0.17$ and $0.15 \mathrm{ppm})$.

Reaction between a and $\mathrm{Et}_{3} \mathrm{SiH}$ (3 equiv) in the presence of 1-B/Al $\left(\mathrm{C}_{6} \mathrm{~F}_{5}\right)_{3}$ (toluene $)_{0.5}\left(\left([\mathrm{~B}]_{0}=\right.\right.$ $[\mathrm{Al}]_{0}=1 \mathrm{~mol} \%$ ) proceeded instantly in $\mathrm{C}_{6} \mathrm{D}_{6}$ at room temperature resulting in $27 \%$ conversion of hydrosilane and formation of $\mathbf{b}$ in $66 \%$ yield (vs hydrosilane).

(a) Stephan, D. W. Frustrated Lewis Pairs: From Concept to Catalysis. Acc. Chem. Res. 2015, 48, 306-316. (b) Voicu, D.; Abolhasani, M.; Choueiri, R.; Lestari, G.; Seiler, C.; Menard, G.; Greener, J.; Guenther, A.; Stephan, D. W.; Kumacheva, E. Microfluidic studies of $\mathrm{CO}_{2}$ sequestration by frustrated Lewis pairs. J. Am. Chem. Soc. 2019, 136, 3875-3880.

Heiden, Z. M.; Lathem, A. P. Establishing the hydride Donor Abilities of Main Group Hydrides. Organometallics 2015, 34, 1818-1827.

(a) Horn, M.; Schappele, L. H.; Lang-Wittkowski, G. ; Mayr, H. ; Ofial, A. R. Towards a Comprehensive Hydride Donor Ability Scale. Chem. Eur. J. 2013, 19, 249-263. (b) Xu, J.; 
Krajewski, A.; Niu, Y.; Kiruba, G. S. M.; Lee, J. K. Chem. Sci. 2019, 10, 8002-8008.

Jaseer, E. A. ; Akhtar, M. N. ; Osman, M. ; Al-Shammari, A. ; Oladipo, H. B.; Garces, K.; Fernandez-Alvarez, F. J.; Al-Khattaf, S.; Oro, L. A. Solvent-free iridium-catalyzed $\mathrm{CO}_{2}$ hydrosilylation: experiments and kinetic modeling. Cat. Sci. Technol. 2015, 3, 274-279.

Blackwell, J. M.; Piers, W. E.; Parvez, M.; McDonald, R. Solution and Solid-State Characteristics of Imine Adducts with Tris(pentafluorophenyl)borane. Organometallics 2002, $21,1400-1407$.

Methylation of secondary aniline $\mathbf{f}$ to tertiary $\mathbf{g}$ can be achieved with $\mathrm{CO}_{2}$ /hydrosilane under mild conditions in the presence of $\mathrm{B}\left(\mathrm{C}_{6} \mathrm{~F}_{5}\right)_{3}$, see: (a) Yang, Z.; Yu, B.; Zhang, H.; Zhao, Y.; Ji, G.; Ma, Z.; Liu, Z. $\mathrm{B}\left(\mathrm{C}_{6} \mathrm{~F}_{5}\right)_{3}$-catalyzed methylation of amines using $\mathrm{CO}_{2}$ as $\mathrm{C} 1$ building block. Green Chemistry 2015, 17, 4189-4193. (b) Tlili, A.; Blondiaux, E.; Frogneux, X.; Cantat; T. Reductive functionalization of $\mathrm{CO}_{2}$ with amines: an entry to formamide, formamidine and methylamine derivatives. Green Chem. 2015, 17, 157-168. (c) Fang, C.; Lu, C.; Liu, M.; Zhu, Y.; Fu, Y.; Lin, B.-L. Selective Formylation and Methylation of Amines using Carbon Dioxide and Hydrosilane Catalyzed by Alkali-Metal Carbonates. ACS Catal. 2016, 6, 7876-7881.

Compound 1-SiEt 3 was independently prepared in $42 \%$ yield (see Experimental Section for details) and characterized by ${ }^{1} \mathrm{H},{ }^{19} \mathrm{~F}\left\{{ }^{1} \mathrm{H}\right\},{ }^{13} \mathrm{C}\left\{{ }^{1} \mathrm{H}\right\}$ NMR spectroscopy (Figures S18, S20 and S21, respectively). Unexpectedly, the stoichiometric reactions of $\mathbf{1}-\mathbf{S i E t}_{3}$ with $\mathrm{B}\left(\mathrm{C}_{6} \mathrm{~F}_{5}\right)_{3}$, attempted under various conditions, failed to provide pure $\left\{\mathbf{B}\left(\mathbf{C}_{6} \mathbf{F}_{5}\right)_{3}\right\} \cdot \mathbf{1}-\mathbf{S i E t}_{3}$ and all resulted in intractable mixtures of products.

Free $\mathrm{B}\left(\mathrm{C}_{6} \mathrm{~F}_{5}\right)_{3}$ and mixtures of unidentified compounds were systematically observed by ${ }^{19} \mathrm{~F}$ NMR spectroscopy. Also, no conclusive data could be obtained from ${ }^{11} \mathrm{~B}$ and ${ }^{29} \mathrm{Si}$ NMR 
spectroscopic studies.

Generation of an intermediary hydrido complex $\left(\mathrm{Me}_{2} \mathrm{~N}\right)_{2} \mathrm{AlH}$ from $\mathrm{Al}\left(\mathrm{NMe}_{2}\right)_{3}$ and $\mathrm{PhSiH}_{3}$ was postulated, see: Allen, L. K.; Garcia-Rodriguez, R.; Wright, D. S. Stoichiometric and catalytic Si-N bond formation using the p-block base $\mathrm{Al}\left(\mathrm{NMe}_{2}\right)_{3}$. Dalton Trans. 2015, 44, 12112-12118.

(a) Parks, D. J.; Blackwell, J. M.; Piers, W. E. Studies on the Mechanism of $\mathrm{B}\left(\mathrm{C}_{6} \mathrm{~F}_{5}\right)_{3}$-Catalyzed Hydrosilation of Carbonyl Functions. J. Org. Chem. 2000, 65, 3090-3098. (b) Piers, W. E.; Marwitz, A. J. V.; Mercier, L. G. Mechanistic Aspects of Bond Activation with Perfluoroarylboranes. Inorg. Chem. 2011, 50, 12252-12262.

Nikonov, G. I.; Vyboishchikov, S. F.; Shirobokov, O. G. Facile Activation of H-H and Si-H Bonds by Boranes. J. Am. Chem. Soc. 2012, 134, 5488-5491.

(a) Parks, D. J.; von Spence, R. E.; Piers, W. E. Bis(pentafluorophenyl)borane: Synthesis, Properties, and Hydroboration Chemistry of a Highly Electrophilic Borane Reagent. Angew. Chem. Int. Ed. 1995, 34, 809-811. (b) Parks, D. J.; Piers, W. E.; Yap G. P. A. Synthesis, Properties, and Hydroboration Activity of the Highly Electrophilic Borane Bis(pentafluorophenyl)borane, $\mathrm{HB}\left(\mathrm{C}_{6} \mathrm{~F}_{5}\right)_{2}$. Organometallics 1998, 17, 5492-5503. Hagele, G.; Weidenbruch, M. Zusammenhange zwischen chemischen Verschiebungen, Kopplungskonstanten und (p-d) $\pi$-Wechselwikungen. Chem. Ber. 1973, 106, 460-470. 1963, 212.

Chen, M. C.; Roberts, J. A. S.; Marks, T. J. New mononuclear and polynuclear perfluoroarylmetatate cocatalysts for stereospecific olefin polymerization. Organometallics 
2004, 23, 932-935.

Zheng, J.; Chevance, S.; Darcel, C.; Sortais, J.-B. Selective reduction of carboxylic acids to aldehydes through manganese catalysed hydrosilylation. Chem. Commun. 2013, 49, 10010-10012.

Maringgele, W.; Meller, A. Synthese von Silylamident und deren Umsetzung mit Boran-derivaten. Z. Anorg. Allg. Chem. 1978, 445, 107-121.

Frisch, M. J.; Trucks, G. W.; Schlegel, H. B.; Scuseria, G. E.; Robb, M. A.; Cheeseman, J. R.; Scalmani, G.; Barone, V.; Mennucci, B.; Petersson, G. A.; Nakatsuji, H.; Caricato, M.; Li, X.; Hratchian, H. P.; Izmaylov, A. F.; Bloino, J.; Zheng, G.; Sonnenberg, J. L.; Hada, M.; Ehara, M.; Toyota, K.; Fukuda, R.; Hasegawa, J.; Ishida, M.; Nakajima, T.; Honda, Y.; Kitao, O.; Nakai, H.; Vreven, T.; Montgomery, J. A., Jr.; Peralta, J. E.; Ogliaro, F.; Bearpark, M.; Heyd, J. J.; Brothers, E.; Kudin, K. N.; Staroverov, V. N.; Kobayashi, R.; Normand, J.; Raghavachari, K.; Rendell, A.; Burant, J. C.; Iyengar, S. S.; Tomasi, J.; Cossi, M.; Rega, N.; Millam, J. M.; Klene, M.; Knox, J. E.; Cross, J. B.; Bakken, V.; Adamo, C.; Jaramillo, J.; Gomperts, R.; Stratmann, R. E.; Yazyev, O.; Austin, A. J.; Cammi, R.; Pomelli, C.; Ochterski, J. W.; Martin, R. L.; Morokuma, K.; Zakrzewski, V. G.; Voth, G. A.; Salvador, P.; Dannenberg, J. J.; Dapprich, S.; Daniels, A. D.; Farkas, Ö.; Foresman, J. B.; Ortiz, J. V.; Cioslowski, J.; Fox, D. J. Gaussian 09, Revision D.01; Gaussian Inc.: Pittsburgh, PA, 2009.

(a) Becke, A. D. Density-functional exchange-energy approximation with correct asymptotic behavior. Phys. Rev. A 1988, 38, 3098. (b) Becke, A. D. Density - functional thermochemistry. III. The role of exact exchange. J. Chem. Phys. 1993, 98, 5648. 

electron density and on a continuum model of the solvent defined by the bulk dielectric constant and atomic surface tensions. J. Phys. Chem. B, 2009, 113, 6378. Phys. 1989, 90, 2154.

Reed, A. E.; Curtiss, L. A.; Weinhold, F. Intermolecular interactions from a natural bond orbital, donor-acceptor viewpoint. Chem. Rev. 1988, 88, 899-926.

(a) G. M. Sheldrick, SHELXS-97, Program for the Determination of Crystal Structures, University of Goettingen (Germany), 1997; (b) G. M. Sheldrick, SHELXL-97, Program for the Refinement of Crystal Structures, University of Goettingen (Germany), 1997. (c) Sheldrick, G. M. Acta. Cryst. 2008, A64, 112-122. 